\title{
Effectiveness of Interventions to Reduce Potentially Inappropriate Medication in Older Patients: A Systematic Review
}

\begin{abstract}
Daniela A. Rodrigues ${ }^{1}$, Ana I. Plácido ${ }^{1}$, Ramona Mateos-Campos ${ }^{2}$, Adolfo Figueiras ${ }^{3,4,5}$, Maria Teresa Herdeiro ${ }^{6}$ and Fátima Roque ${ }^{1,7 *}$

${ }^{1}$ Research Unit for Inland Development, Polytechnic Institute of Guarda (UDI-IPG), Guarda, Portugal, ${ }^{2}$ Area of Preventive Medicine and Public Health, Department of Biomedical and Diagnostic Sciences, University of Salamanca, Salamanca, Spain, ${ }^{3}$ Department of Preventive Medicine and Public Health, University of Santiago de Compostela, Santiago de Compostela, Spain, ${ }^{4}$ Consortium for Biomedical Research in Epidemiology and Public Health (CIBER Epidemiology and Public Health-CIBERESP), Madrid, Spain, ${ }^{5}$ Health Research Institute of Santiago de Compostela (IDIS), Santiago de Compostela, Spain, ${ }^{6}$ Department of Medical Sciences, Institute of Biomedicine (iBiMED), University of Aveiro, Aveiro, Portugal, ${ }^{7}$ Health Sciences Research Centre, University of Beira Interior (CICS-UBI), Covilhã, Portugal
\end{abstract}

OPEN ACCESS

Edited by:

Elisabetta Poluzzi,

University of Bologna, Italy

Reviewed by:

Anna Birna Almarsdóttir, University of Copenhagen, Denmark

Carlotta Lunghi,

Université du Québec à Rimouski,

Canada

*Correspondence: Fátima Roque froque@ipg.pt

Specialty section:

This article was submitted to Pharmacoepidemiology,

a section of the journal

Frontiers in Pharmacology

Received: 15 September 2021 Accepted: 12 November 2021

Published: 24 January 2022

Citation:

Rodrigues DA, Plácido Al, Mateos-Campos R, Figueiras A, Herdeiro MT and Roque F (2022) Effectiveness of Interventions to Reduce Potentially Inappropriate Medication in Older Patients: A Systematic Review. Front. Pharmacol. 12:777655. doi: 10.3389/fphar.2021.777655
Background: Age-related multiple comorbidities cause older adults to be prone to the use of potentially inappropriate medicines (PIM) resulting in an increased risk of adverse events. Several strategies have emerged to support PIM prescription, and a huge number of interventions to reduce PIM have been proposed. This work aims to analyze the effectiveness of PIM interventions directed to older adults.

Methods: A systematic review was performed searching the literature in the MEDLINE PubMed, EMBASE, and Cochrane scientific databases for interventional studies that assessed the PIM interventions in older adults ( $\geq 65$ years).

Results: Forty-seven articles were included, involving 52 to 124,802 patients. Various types of interventions were analyzed such as medication review, educational strategies, clinical decision support system, and organizational and multifaceted approaches. In the hospital, the most successful intervention was medication review $(75.0 \%)$, while in primary care, the analysis of all included studies revealed that educational strategies were the most effective. However, the analysis of interventions that have greater evidence by its design was inconclusive.

Conclusion: The results obtained in this work suggested that PIM-setting-directed interventions should be developed to promote the wellbeing of the patients through PIM reduction. Although the data obtained suggested that medication review was the most assertive strategy to decrease the number of PIM in the hospital setting, more studies are necessary.

Systematic Review Registration: [https://www.crd.york.ac.uk/prospero/display_ record.php?ID=CRD42021233484], identifier [PROSPERO 2021 CRD42021233484].

Keywords: review, potentially inappropriate medication, interventions, effectiveness, older adults 


\section{INTRODUCTION}

The increase in life expectancy associated with a declined birth rate contributed to rapid population aging (United Nations, 2019). Even though the world population is getting older, aging populations differ by region and level of development (Beard et al., 2016). Globally, it is estimated that in 2050 the number of older adults will reach 1.5 billion and will outnumber adolescents and youth aged 15-24 years (1.3 billion) (United Nations, 2019).

Considering that more than half of older adults have at least two chronic diseases (Barnett et al., 2012), these societal transformations pose a significant challenge in health systems and increase the consumption of health resources, including medicines. In addition, the treatment of chronic diseases is based on single disease-centered guidelines that can lead to an overwhelming of medication, and polypharmacy can easily occur (Barnett et al., 2012). Age-related pharmacokinetic and pharmacodynamic alterations associated with the use of multiple medicines can potentiate the consumption of potentially inappropriate medications (PIM) and facilitate the occurrence of adverse drug reactions (ADR) in frail older adults (Motter et al., 2018; Hefner et al., 2021).

PIM is defined as medicines that should not be prescribed because the risk of adverse events outweighs the clinical benefit, especially when more effective alternatives are available (RenomGuiteras et al., 2015). The prescription of PIM has received special attention from the health community, and interventions aim to optimize medication prescribing and increase the benefit/risk ratio associated with the patients (Anderson et al., 1997; Simonson and Feinberg, 2005). In the last decades, several studies have been done to evaluate the effectiveness of PIM interventions in primary care, such as in hospitals and nursing homes. Nevertheless, the studies display widely differing methodology and inconsistent results, and to our knowledge, there are no systematic reviews comparing the effectiveness of different kinds of interventions.

Thus, this study aims to critically review the effectiveness of interventions to reduce PIM prescriptions in older adults.

\section{METHODS}

This systematic review followed the PRISMA (Preferred Reporting Items for Systematic Reviews and Meta-Analyses) 2020 guidelines (Page et al., 2021) (Supplementary Table S1). The research protocol is registered on PROSPERO (CRD42021233484).

\subsection{Search Strategy}

A literature search was conducted in January 2021 and updated in February 2021 on the MEDLINE PubMed, EMBASE. A search was also conducted in the Cochrane database in October 2021. The search strategy was designed to identify relevant studies addressing interventions on PIM prescriptions in older adults, using the following broad-based search terms strategy: "(elderly OR "elderly patient" OR "older patient ${ }^{\star}$ ” OR "older adult ${ }^{\star}$ ” OR "geriatric patient*") AND (PIM OR PIP OR "potentially inappropriate medicine" OR "potentially inappropriate medication" OR "potentially inappropriate prescribing" OR "prescribing patterns" OR "Prescription Drug Misuse" OR "Prescription Drug Overuse" OR “deprescri*” OR "potentially inappropriate prescription ${ }^{\star}$ ) AND (prevention OR reduction OR decrease OR impact) AND (intervention OR trial).”

\subsection{Selection Criteria}

This systematic review included the following: 1) all studies focused on PIM interventions directed to older adults ( $\geq 65$ years) that aimed to optimize their pharmacotherapy; 2 ) controlled intervention studies and case series studies; and 3) all studies published in Portuguese, English, or Spanish between January 1, 2000, and December 31, 2020.

Excluded from this work were reviews, meta-analyses, opinions, letters to the editor that do not provide original data, comments, reports, studies addressing PIM in a specific pathology, and studies targeting a limited and predefined class of PIM.

\subsection{Outcomes Measures}

Our primary outcome measure was the effectiveness of the PIM interventions through the analysis of the change rate between the mean number of PIM per patient and/or the mean number of patients with PIM before and after an intervention.

\subsection{Data Extraction}

Two researchers (AP and DR) independently screened all titles and abstracts retrieved from the databases accordingly with the inclusion criteria. To evaluate the eligibility of full-text articles, two researchers (AP and DR) independently screened the full text of the articles. All discrepancies were resolved through discussion with the help of a third researcher (FR).

\subsection{Quality Assessment}

Two researchers (AP and DR) independently evaluated the quality and susceptibility to the bias of the included studies using the "Quality Assessment of Controlled Intervention Studies" and the "Quality Assessment Tool for Case Series Studies" tools, depending on study design (National Heart Laboratory, 2013). All discrepancies were resolved through discussion with a third (FR) or fourth researcher $(\mathrm{MH})$.

\subsection{Data Synthesis and Presentation}

Two researchers (AP and DR) independently extracted data from the included studies. The data extracted from each article include authors, publication year, study design, country, sample size, patients' age, type of intervention applied, PIM screening tool, outcome measures, and main results.

To better analyze the extracted data, studies were grouped according to the intervention used. Within the intervention used, studies were grouped according to the setting where the intervention occurs. Five different interventions were identified in the included studies, and their descriptions were based on the following pre-defined definitions: 1) Medication review: "a structured evaluation of a patient's medicines to optimize 


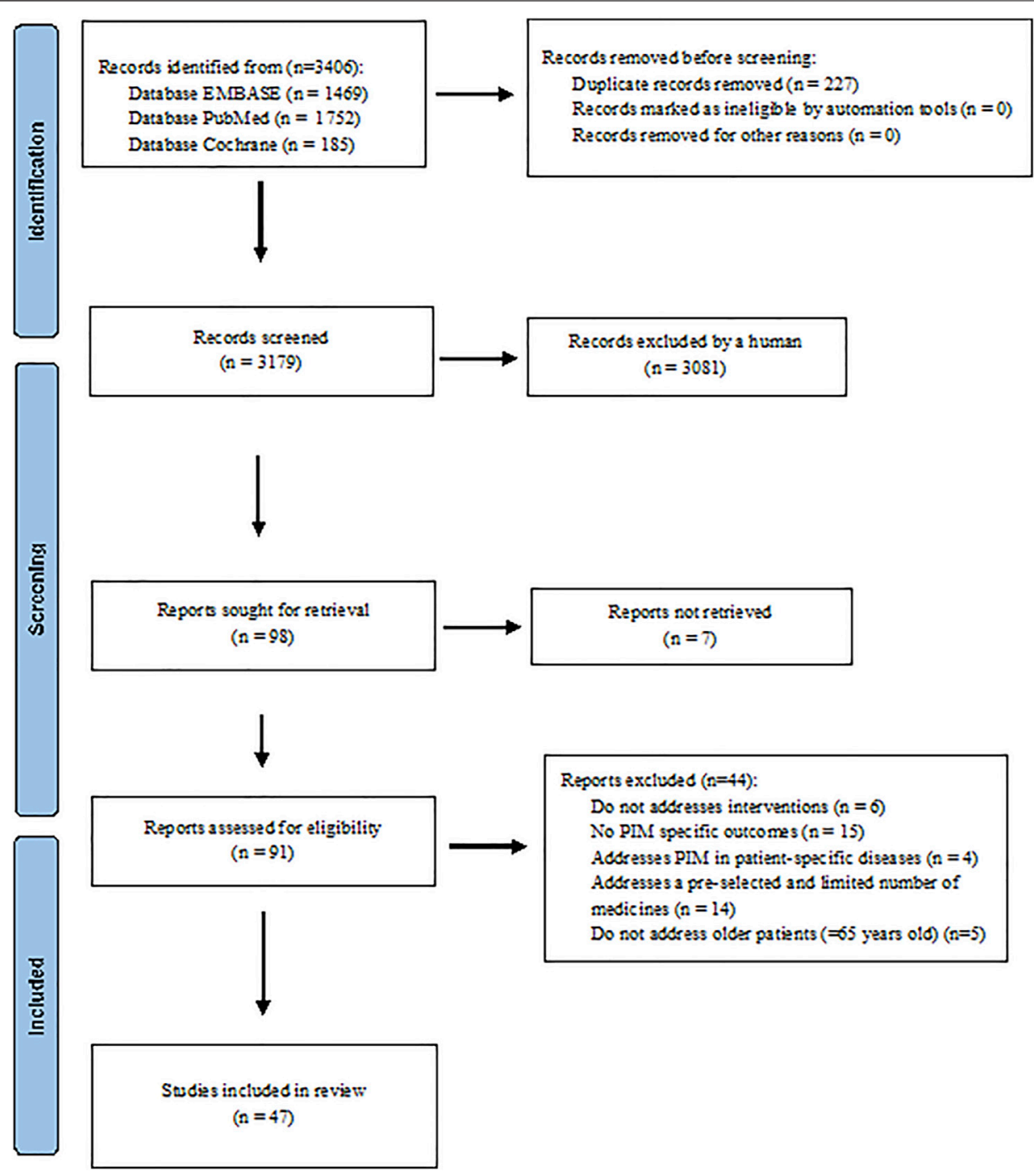

FIGURE 1 | PRISMA diagram of the literature selection in this systematic review.

medicines use and improve health outcomes. This entails detecting drug-related problems and recommending interventions" (Griese-Mammen et al., 2018); 2) educational interventions: "a package of interventions aimed to refresh the basic pharmacology competencies of a healthcare professional to change the prescription. The approaches used in the educational interventions included: interactive teaching, mailed educational material combined with individual feedback, and face-to-face visits to physicians" (Kaur et al., 2009); 3) clinical decision support systems (CDSS): "electronic tools that prompt provider behaviors in various areas of patient care, including medication ordering, chronic disease management, health care screening, and vaccination. CDSS can provide physicians, nurses, pharmacists, and other care providers with patient-specific prompts or warnings, treatment guidelines (e.g., order sets), automatic medication dosing calculators, or reports of overdue tests and medications as appropriate" (Bhugra and Cutter, 2001);
4) multifaceted interventions: "any intervention including two or more components." In this study we classified as a multifaceted approach studies that used a combination of the interventions described above (Squires et al., 2014); and 4) organizational strategies: a combination of methodologies to improve the quality indicators. This type of intervention can include several methodologies, such as diagnostic activity, team building, intergroup relationship, sensitivity training, etc. In this work, organizational strategies include showing charts with the percentage of patients with PIM, an educational session with practices to identify patients with PIM, and frequent reunions to evaluate PIM indicators (Cady and Kim, 2017).

\subsection{Statistical Analysis}

A qualitative analysis was done if at least two studies had comparable outcomes. The heterogeneity of the studies was 
assessed through the comparison between the interventions. The efficacy of the interventions was presented as a change rate between the mean number of PIM per patient and/or the mean number of patients with PIM before and after an intervention.

\section{RESULTS}

\subsection{Study Selection}

The search of the databases yielded 3,406 citations (Figure 1). After screening titles and abstracts, 98 articles potentially met the inclusion criteria. Because seven articles were not retrieved, only 91 articles were fully screened. Among these, 42 were excluded because they did not address interventions $(n=6)$, did not report PIM specific outcomes $(n=15)$, addressed PIM in patientspecific diseases $(n=4)$, addressed a pre-selected and/or a limited number of medicines $(n=14)$, and did not address older patients ( $\geq 65$ years old) $(n=5)$ (Supplementary Table S2).

Forty-seven articles (Allard et al., 2001; Brown and Earnhart, 2004; Fick et al., 2004; Spinewine et al., 2007; Wessell et al., 2008; Castelino et al., 2010; Lampela et al., 2010; Gallagher et al., 2011; Keith et al., 2013; Rognstad et al., 2013; Dalleur et al., 2014; Franchi et al., 2014, 2016; Frankenthal et al., 2014; Lopatto et al., 2014; Clyne et al., 2015, 2016; Ilić et al., 2015; Tallon et al., 2015; Campins et al., 2016; Moss et al., 2016; Urfer et al., 2016; Frankenthal et al., 2017; Price et al., 2017; Stevens et al., 2017; Van der Linden et al., 2017; Vanderman et al., 2017; Chan et al., 2018; Etxeberria et al., 2018; Fajreldines et al., 2018; Gibert et al., 2018; Hurmuz et al., 2018; Najjar et al., 2018; Sennesael et al., 2018; Stuckey et al., 2018; Van Der Linden et al., 2018; Vandenberg et al., 2018; Boersma et al., 2019; GutiérrezValencia et al., 2019; Khera et al., 2019; Liu et al., 2019; McDonald et al., 2019; Moss et al., 2019; Regueiro et al., 2019; Vu and Huong, 2019; Akkawi et al., 2020; Winata et al., 2020) fulfilled the inclusion criteria and were included in this systematic review (Figure 1).

\subsection{Characteristics of Included Studies}

A description of the characteristics of the included studies is presented in Table 1. Among the included studies, 24 were conducted in Europe (Spinewine et al., 2007; Lampela et al., 2010; Gallagher et al., 2011; Keith et al., 2013; Rognstad et al., 2013; Dalleur et al., 2014; Franchi et al., 2014; Lopatto et al., 2014; Clyne et al., 2015; Ilić et al., 2015; Tallon et al., 2015; Campins et al., 2016; Clyne et al., 2016; Franchi et al., 2016; Urfer et al., 2016; Van der Linden et al., 2017; Etxeberria et al., 2018; Gibert et al., 2018; Hurmuz et al., 2018; Sennesael et al., 2018; Van Der Linden et al., 2018; Boersma et al., 2019; Gutiérrez-Valencia et al., 2019; Regueiro et al., 2019), 14 in North America (Allard et al., 2001; Brown and Earnhart, 2004; Fick et al., 2004; Wessell et al., 2008; Moss et al., 2016; Price et al., 2017; Stevens et al., 2017; Vanderman et al., 2017; Chan et al., 2018; Stuckey et al., 2018; Vandenberg et al., 2018; Khera et al., 2019; McDonald et al., 2019; Moss et al., 2019), six in Asia (Frankenthal et al., 2014, 2017; Najjar et al., 2018; Liu et al., 2019; Vu and Huong, 2019; Akkawi et al., 2020), two in Oceania (Castelino et al., 2010; Winata et al.,
2020), and one in South America (Fajreldines et al., 2018). The most frequented settings of the included studies were hospital $(n=26)$ (Brown and Earnhart, 2004; Spinewine et al., 2007; Gallagher et al., 2011; Dalleur et al., 2014; Franchi et al., 2014; Tallon et al., 2015; Franchi et al., 2016; Moss et al., 2016; Urfer et al., 2016; Stevens et al., 2017; Van der Linden et al., 2017; Vanderman et al., 2017; Chan et al., 2018; Fajreldines et al., 2018; Najjar et al., 2018; Sennesael et al., 2018; Van Der Linden et al., 2018; Vandenberg et al., 2018; Gutiérrez-Valencia et al., 2019; Liu et al., 2019; McDonald et al., 2019; Moss et al., 2019; Regueiro et al., 2019; Vu and Huong, 2019; Akkawi et al., 2020; Winata et al., 2020) and primary care $(n=18)$ (Allard et al., 2001; Fick et al., 2004; Wessell et al., 2008; Castelino et al., 2010; Lampela et al., 2010; Keith et al., 2013; Rognstad et al., 2013; Lopatto et al., 2014; Clyne et al., 2015; Campins et al., 2016; Clyne et al., 2016; Price et al., 2017; Etxeberria et al., 2018; Gibert et al., 2018; Hurmuz et al., 2018; Stuckey et al., 2018; Boersma et al., 2019; Khera et al., 2019). The number of participants in the studies ranged from 52 to 124,802 . In the included studies, the average age of the participants ranged from 71 to 88.4 years. However, 12 studies did not report an average age of patients, although all of these studies provided an age range: 10 studies included older adults aged $\geq 65$ years (Fick et al., 2004; Wessell et al., 2008; Moss et al., 2016; Frankenthal et al., 2017; Price et al., 2017; Stevens et al., 2017; Najjar et al., 2018; Vandenberg et al., 2018; Moss et al., 2019; Vu and Huong, 2019), one study included patients aged $\geq 70$ years (Rognstad et al., 2013), and one study included patients aged $\geq 75$ years (Lampela et al., 2010).

\subsection{Quality of Included Studies}

The quality assessment tools results of each study are reported in Table 2. Twenty-five articles fulfilled more than $80 \%$ of the exploratory questions (Castelino et al., 2010; Gallagher et al., 2011; Rognstad et al., 2013; Frankenthal et al., 2014; Lopatto et al., 2014; Clyne et al., 2015, 2016; Campins et al., 2016; Price et al., 2017; Stevens et al., 2017; Vanderman et al., 2017; Chan et al., 2018; Etxeberria et al., 2018; Fajreldines et al., 2018; Gibert et al., 2018; Hurmuz et al., 2018; Sennesael et al., 2018; GutiérrezValencia et al., 2019; Khera et al., 2019; Liu et al., 2019; Moss et al., 2019; Regueiro et al., 2019; Vu and Huong, 2019; Akkawi et al., 2020; Winata et al., 2020). Eighteen studies pointed out clearly potential sources of bias (Brown and Earnhart, 2004; Fick et al., 2004; Spinewine et al., 2007; Wessell et al., 2008; Lampela et al., 2010; Rognstad et al., 2013; Campins et al., 2016; Franchi et al., 2016; Urfer et al., 2016; Frankenthal et al., 2017; Price et al., 2017; Van der Linden et al., 2017; Etxeberria et al., 2018; Gibert et al., 2018; Boersma et al., 2019; Gutiérrez-Valencia et al., 2019; Khera et al., 2019; Moss et al., 2019). Through analysis of Table 2, the main limitations were related to the low sample size and the lack of blinded intervention. Besides that, most of the studies did not do or report follow-up results, so it is not possible to understand if the interventions were effective in the middle/long-term.

\subsection{Evidence of Effectiveness}

\subsubsection{PIM Screening Tools}

Thirty-seven studies used validated and published criteria to identify PIM, including Beers criteria $(n=16)$ (Brown and 
TABLE 1 | Characteristics of the included studies $(n=47)$.

\begin{tabular}{|c|c|c|c|c|c|c|c|}
\hline \multirow[t]{2}{*}{ Author (year) } & \multirow[t]{2}{*}{ Country } & \multirow[t]{2}{*}{ Study design } & \multirow[t]{2}{*}{ Setting } & \multicolumn{2}{|c|}{ Elderly patients' sample } & \multirow[t]{2}{*}{ Comparator } & \multirow{2}{*}{$\begin{array}{l}\text { Quality } \\
\text { assessment/ } \\
\text { (score } \\
\text { obtained/total } \\
\text { score) }\end{array}$} \\
\hline & & & & Sample size & $\begin{array}{l}\text { Mean age } \\
\text { (SD or } \\
\text { IQR) }\end{array}$ & & \\
\hline Akkawi et al. (2020) & Malaysia & Case series & Hospital & $\begin{array}{l}\text { B: } 240 \\
\text { A: } 240\end{array}$ & $\begin{array}{l}\text { B: } 71.9(5.8) \\
\text { A: } 72.9(5.7)\end{array}$ & Baseline & $8 / 9^{a}$ \\
\hline Winata et al. (2020) & Australia & Case series & $\begin{array}{l}\text { Hospital (aged care } \\
\text { wards) }\end{array}$ & $\begin{array}{l}\text { B: } 121 \\
\text { A: } 107\end{array}$ & $\begin{array}{l}\text { B: } 83.9(7.2) \\
\text { A: } 83.3(7.2)\end{array}$ & Baseline & $8 / 9^{a}$ \\
\hline $\begin{array}{l}\text { Boersma et al. } \\
\text { (2019) }\end{array}$ & Netherlands & $\mathrm{RCT}$ & $\begin{array}{l}\text { Geriatric clinic } \\
\text { (outpatients) }\end{array}$ & $\begin{array}{l}\text { C: } 59 \\
\text { I: } 65\end{array}$ & $\begin{array}{l}\text { C: } 79.0(6.0) \\
\text { I: } 77.8(5.7)\end{array}$ & Usual care & $10 / 14^{b}$ \\
\hline $\begin{array}{l}\text { Gutiérrez-Valencia } \\
\text { et al. (2019) }\end{array}$ & Spain & Prospective study & $\begin{array}{l}\text { Tertiary public hospital } \\
\text { (acute geriatric unit) }\end{array}$ & 234 & $87.6(4.6)$ & Baseline & $8 / 9^{a}$ \\
\hline Khera et al. (2019) & Canada & $\begin{array}{l}\text { Quasi-experimental } \\
\text { pretest-posttest }\end{array}$ & $\begin{array}{l}\text { Primary care } \\
\text { (community-dwelling } \\
\text { patients) }\end{array}$ & 54 & $\begin{array}{c}81.7(6.74 \\
65-95)\end{array}$ & $\begin{array}{l}\text { Before medication } \\
\text { review }\end{array}$ & $8 / 9^{a}$ \\
\hline Liu et al. (2019) & Taiwan & Interventional & $\begin{array}{l}\text { Tertiary medical center } \\
\text { (emergency department) }\end{array}$ & $\begin{array}{l}B: 243 \\
A: 668\end{array}$ & $\begin{array}{l}\text { B: } 78.2(7.7) \\
A: 78.1(7.7)\end{array}$ & $\begin{array}{l}\text { Before } \\
\text { implementation of } \\
\text { the intervention }\end{array}$ & $8 / 9^{a}$ \\
\hline $\begin{array}{l}\text { McDonald et al. } \\
\text { (2019) }\end{array}$ & Canada & $\begin{array}{l}\text { Non-randomized } \\
\text { controlled before and } \\
\text { after study }\end{array}$ & $\begin{array}{l}\text { Medical clinical teaching } \\
\text { units (internal medicine } \\
\text { department) }\end{array}$ & $\begin{array}{l}\text { C: } 383 \\
\text { I: } 417\end{array}$ & $\begin{array}{l}\text { C: } 79 \text { (73-86) } \\
\text { I: } 81(74-88)\end{array}$ & Usual care & $8 / 14^{b}$ \\
\hline Moss et al. (2019) & United States & Case series & $\begin{array}{l}\text { Veteran Affairs Medical } \\
\text { Center (emergency } \\
\text { department) }\end{array}$ & $\begin{array}{l}\mathrm{C}: 2,500 \\
\mathrm{I}: 3,162\end{array}$ & $\geq 65$ & Untrained cohort & $8 / 9^{a}$ \\
\hline Regueiro et al. (2019) & Spain & $\begin{array}{l}\text { Quasi-experimental } \\
\text { pre-post }\end{array}$ & $\begin{array}{l}\text { University hospital } \\
\text { (internal medicine } \\
\text { department) }\end{array}$ & 174 & $82.6(6.9)$ & $\begin{array}{l}\text { Before } \\
\text { implementation of } \\
\text { the intervention }\end{array}$ & $8 / 9^{a}$ \\
\hline Vu and Huong (2019) & Vietnam & Case series & $\begin{array}{l}\text { General hospital } \\
\text { (endocrinology, } \\
\text { cardiology, and } \\
\text { neurology departments) }\end{array}$ & $\begin{array}{l}\text { B: } 211 \\
\text { A: } 208\end{array}$ & $\geq 65$ & Baseline & $8 / 9^{a}$ \\
\hline Chan et al. (2018) & Canada & $\begin{array}{l}\text { Retrospective single- } \\
\text { center pre-post } \\
\text { cohort }\end{array}$ & $\begin{array}{l}\text { Tertiary hospital (acute } \\
\text { care unit) }\end{array}$ & $\begin{array}{l}B: 70 \\
A: 67\end{array}$ & $\begin{array}{l}B: 88.1(4.3) \\
A: 88.4(5.1)\end{array}$ & $\begin{array}{l}\text { Before } \\
\text { implementation of } \\
\text { the intervention }\end{array}$ & $7 / 9^{a}$ \\
\hline $\begin{array}{l}\text { Etxeberria et al. } \\
\text { (2018) }\end{array}$ & Spain & Case series & Primary health care & 503 & $84.9(3.8)$ & $\begin{array}{l}\text { Before } \\
\text { implementation of } \\
\text { the intervention }\end{array}$ & $7 / 9^{a}$ \\
\hline $\begin{array}{l}\text { Fajreldines et al. } \\
(2018)\end{array}$ & Argentina & Case series & Hospital & $\begin{array}{l}\text { B: } 640 \\
\text { A: } 622\end{array}$ & $\begin{array}{l}\text { B: } 80.9(9.8) \\
\text { A: } 79.3(9.7)\end{array}$ & $\begin{array}{l}\text { Before } \\
\text { implementation of } \\
\text { the intervention }\end{array}$ & $8 / 9^{a}$ \\
\hline Hurmuz et al. (2018) & Netherlands & $\begin{array}{l}\text { Retrospective } \\
\text { longitudinal pretest } \\
\text { vs. posttest }\end{array}$ & Community pharmacy & 126 & $76.0(7.4)$ & $\begin{array}{l}\text { Before } \\
\text { implementation of } \\
\text { the intervention }\end{array}$ & $8 / 9^{a}$ \\
\hline Naijar et al. (2018) & Saudi Arabia & $\begin{array}{l}\text { Prospective pretest } \\
\text { vs. posttest design }\end{array}$ & Hospital & $\begin{array}{l}\text { B: } 200 \\
\text { A: } 200\end{array}$ & $\geq 65$ & Baseline & $6 / 9^{a}$ \\
\hline Gibert et al. (2018) & France & - & Primary care & 172 & $83.5(4.9)$ & $\begin{array}{l}\text { Before } \\
\text { implementation of } \\
\text { the intervention }\end{array}$ & $8 / 9^{a}$ \\
\hline $\begin{array}{l}\text { Sennesael et al. } \\
\text { (2018) }\end{array}$ & Belgium & $\begin{array}{l}\text { Retrospective } \\
\text { interrupted time } \\
\text { series study }\end{array}$ & $\begin{array}{l}\text { Teaching hospital } \\
\text { (geriatric unit) }\end{array}$ & 120 & $85(81-88)^{c}$ & $\begin{array}{l}\text { Standard geriatric } \\
\text { care }\end{array}$ & $7 / 9^{a}$ \\
\hline Stuckey et al. (2018) & United States & $\begin{array}{l}\text { Prospective quality } \\
\text { improvement project }\end{array}$ & $\begin{array}{l}\text { Family medicine clinic } \\
\text { (residency training } \\
\text { outpatients) }\end{array}$ & 34 & $74(5)$ & $\begin{array}{l}\text { Before } \\
\text { implementation of } \\
\text { the intervention }\end{array}$ & $5 / 9^{a}$ \\
\hline $\begin{array}{l}\text { Vandenberg et al. } \\
\text { (2018) }\end{array}$ & United States & $\begin{array}{l}\text { Quality improvement } \\
\text { program }\end{array}$ & $\begin{array}{l}\text { Veteran Affairs Medical } \\
\text { Center (community- } \\
\text { based outpatient clinic) }\end{array}$ & $>7,000$ & $\geq 65$ & $\begin{array}{l}\text { Before } \\
\text { implementation of } \\
\text { the intervention }\end{array}$ & $3 / 14^{b}$ \\
\hline $\begin{array}{l}\text { Van Der Linden et al. } \\
\text { (2018) }\end{array}$ & Belgium & Case series & Teaching hospital & $\begin{array}{l}\text { B: } 29 \\
\text { A: } 30\end{array}$ & $\begin{array}{l}\text { B: } 83\left(79-86^{c}\right. \\
\text { A: } 83(78-88)^{c}\end{array}$ & Usual care & $8 / 14^{b}$ \\
\hline $\begin{array}{l}\text { Frankenthal et al. } \\
\text { (2017) }\end{array}$ & Israel & $\mathrm{RCT}$ & $\begin{array}{l}\text { Chronic care geriatric } \\
\text { facility }\end{array}$ & $\begin{array}{l}\text { C: } 126 \\
\text { I: } 126\end{array}$ & $\geq 65$ & Usual care & $7 / 14^{\mathrm{b}}$ \\
\hline Price et al. (2017) & Canada & $\mathrm{RCT}$ & Primary care & $\begin{array}{l}\text { C: } 1,086 \\
\text { I: } 1,204\end{array}$ & $\geq 65$ & Baseline rate & $11 / 14^{b}$ \\
\hline
\end{tabular}


TABLE 1 | (Continued) Characteristics of the included studies $(n=47)$.

\begin{tabular}{|c|c|c|c|c|c|c|c|}
\hline \multirow[t]{2}{*}{ Author (year) } & \multirow[t]{2}{*}{ Country } & \multirow[t]{2}{*}{ Study design } & \multirow[t]{2}{*}{ Setting } & \multicolumn{2}{|c|}{ Elderly patients' sample } & \multirow[t]{2}{*}{ Comparator } & \multirow{2}{*}{$\begin{array}{c}\text { Quality } \\
\text { assessment/ } \\
\text { (score } \\
\text { obtained/total } \\
\text { score) }\end{array}$} \\
\hline & & & & Sample size & $\begin{array}{l}\text { Mean age } \\
\text { (SD or } \\
\text { IQR) }\end{array}$ & & \\
\hline Stevens et al. (2017) & United States & - & $\begin{array}{l}\text { Veteran Affairs Medical } \\
\text { Center (emergency } \\
\text { department) }\end{array}$ & - & $\geq 65$ & - & $9 / 9^{a}$ \\
\hline $\begin{array}{l}\text { Vanderman et al. } \\
(2017)\end{array}$ & United States & $\begin{array}{l}\text { Retrospective cohort } \\
\text { study }\end{array}$ & $\begin{array}{l}\text { Veteran Affairs Medical } \\
\text { Center (ambulatory } \\
\text { clinics) }\end{array}$ & $\begin{array}{l}B: 1,539 \\
A: 1,490\end{array}$ & $\begin{array}{l}\text { B: } 71.0(6.72) \\
A: 71.0(6.65)\end{array}$ & Usual care & $7 / 9^{a}$ \\
\hline $\begin{array}{l}\text { Van der Linden et al. } \\
\text { (2017) }\end{array}$ & Belgium & $\begin{array}{l}\text { Prospective } \\
\text { controlled trial }\end{array}$ & $\begin{array}{l}\text { Hospital (acute geriatric } \\
\text { ward) }\end{array}$ & $\begin{array}{l}\text { C: } 81 \\
\text { I: } 91\end{array}$ & $\begin{array}{l}\text { C: } 84.5(4.97) \\
\text { I: } 84.5(4.69)\end{array}$ & Usual care & $8 / 14^{b}$ \\
\hline Campins et al. (2016) & Spain & $\mathrm{RCT}$ & $\begin{array}{l}\text { Primary health care } \\
\text { center }\end{array}$ & $\begin{array}{l}\text { C: } 251 \\
\text { I: } 252\end{array}$ & $\begin{array}{l}\text { C: } 78.78(5.46) \\
\text { I: } 79.16(5.50)\end{array}$ & $\begin{array}{l}\text { Routine clinical } \\
\text { practice }\end{array}$ & $12 / 14^{b}$ \\
\hline Clyne et al. (2016) & Ireland & $\mathrm{RCT}$ & Primary care & $\begin{array}{l}\text { C: } 97 \\
\text { I: } 99\end{array}$ & $\begin{array}{l}\text { C: } 76.4(4.8) \\
\text { I: } 77.1(4.9)\end{array}$ & Usual care & $11 / 14^{b}$ \\
\hline Franchi et al. (2016) & Italy & $\mathrm{RCT}$ & $\begin{array}{l}\text { Hospital (internal } \\
\text { medicine and geriatric } \\
\text { wards) }\end{array}$ & $\begin{array}{l}\text { C: } 350 \\
\text { I: } 347\end{array}$ & $\begin{array}{l}\text { C: } 83.8(5.6) \\
\text { I: } 83.7(5.9)\end{array}$ & Baseline & $9 / 14^{b}$ \\
\hline Moss et al. (2016) & United States & - & $\begin{array}{l}\text { Veteran Affairs Medical } \\
\text { Center (emergency } \\
\text { department) }\end{array}$ & 23,168 & $\geq 65$ & - & $6 / 9^{a}$ \\
\hline Urfer et al. (2016) & Switzerland & Case series & $\begin{array}{l}\text { Hospital (internal } \\
\text { medicine ward) }\end{array}$ & $\begin{array}{l}\text { C: } 450 \\
\text { I: } 450\end{array}$ & $\begin{array}{l}\text { C: } 79 \text { (73-84) } \\
\text { I: } 76(71-83)\end{array}$ & $\begin{array}{l}\text { Patients } \\
\text { hospitalized in some } \\
\text { division }\end{array}$ & $8 / 14^{b}$ \\
\hline Clyne et al. (2015) & Ireland & $\mathrm{RCT}$ & Primary care & $\begin{array}{l}\text { C: } 97 \\
\text { I: } 99\end{array}$ & $\begin{array}{l}\text { C: } 76.4(4.8) \\
\text { I: } 77.1(4.9)\end{array}$ & Usual care & $13 / 14^{b}$ \\
\hline Ilić et al. (2015) & Serbia & Case series & Nursing homes & 104 & $82.6(2.1)$ & $\begin{array}{l}\text { Before } \\
\text { implementation of } \\
\text { the intervention }\end{array}$ & $7 / 9^{a}$ \\
\hline Tallon et al. (2015) & Ireland & Case series & Teaching hospital & $\begin{array}{l}\mathrm{B}: 60 \\
\mathrm{~A}: 48\end{array}$ & $\begin{array}{l}\text { B: } 75(70-80) \\
A: 78(71-83)\end{array}$ & Standard care & $7 / 9^{a}$ \\
\hline Dalleur et al. (2014) & Belgium & $\mathrm{RCT}$ & Teaching hospital & $\begin{array}{l}\text { C: } 72 \\
\text { I: } 74\end{array}$ & $\begin{array}{l}\text { C: } 86 \text { (81-89) } \\
\text { I: } 84(81-87)\end{array}$ & Usual care & $8 / 14^{b}$ \\
\hline Franchi et al. (2014) & Italy & $\mathrm{RCT}$ & $\begin{array}{l}\text { Hospital (internal } \\
\text { medicine ward) }\end{array}$ & $\begin{array}{l}\text { Admission C: } \\
\text { 41; I: } 40 \\
\text { Discharge C: } \\
\text { 33; I: } 37\end{array}$ & $\begin{array}{c}\text { Admission } \\
\text { C85.58 (5.99), I: } \\
82.8 \text { (5.59) } \\
\text { Discharge, C: } \\
80.92 \text { (4.53), I: } \\
82.49(4.82)\end{array}$ & $\begin{array}{l}\text { Only the basic } \\
\text { notions of } \\
\text { pharmacology }\end{array}$ & $9 / 14^{b}$ \\
\hline $\begin{array}{l}\text { Frankenthal et al. } \\
\text { (2014) }\end{array}$ & Israel & $\mathrm{RCT}$ & $\begin{array}{l}\text { Chronic care geriatric } \\
\text { facility }\end{array}$ & $\begin{array}{l}\text { C: } 176 \\
\text { I: } 183\end{array}$ & $82.7(8.7)$ & Usual care & $12 / 14^{b}$ \\
\hline Lopatto et al. (2014) & Italy & - & $\begin{array}{l}\text { Health authority } \\
\text { database }\end{array}$ & 111,282 & $75.29(8.34)$ & - & $7 / 9^{a}$ \\
\hline Keith et al. (2013) & Italy & $\begin{array}{l}\text { Multi-phase } \\
\text { prospective }\end{array}$ & $\begin{array}{l}\text { Parma local health } \\
\text { authority database }\end{array}$ & $\begin{array}{l}\text { C: } 81,597 \\
\text { I: } 78,482\end{array}$ & $\begin{array}{l}\text { C: } 75.6(7.3) \\
\text { I: } 75.4(7.2)\end{array}$ & $\begin{array}{l}\text { Region local health } \\
\text { authority database }\end{array}$ & $6 / 14^{b}$ \\
\hline $\begin{array}{l}\text { Rognstad et al. } \\
\text { (2013) }\end{array}$ & Norway & $\mathrm{RCT}$ & General practice & $\begin{array}{c}\text { Control group } \\
\text { B: } 35,073 \\
\text { After: } 35,211 \\
\text { Intervention } \\
\text { group } \\
\text { B: } 46,737 \\
\text { A: } 45,310\end{array}$ & $\geq 70$ & Baseline data & $11 / 14^{b}$ \\
\hline $\begin{array}{l}\text { Gallagher et al. } \\
\text { (2011) }\end{array}$ & Ireland & $\mathrm{RCT}$ & $\begin{array}{l}\text { Hospital (emergency } \\
\text { department) }\end{array}$ & $\begin{array}{l}\text { C: } 192 \\
\text { I: } 190\end{array}$ & $\begin{array}{c}\text { C: } 77 \\
(71-81.75) \\
\text { I: } 74.5(71-80)\end{array}$ & Usual care & $13 / 14^{b}$ \\
\hline $\begin{array}{l}\text { Castelino et al. } \\
\text { (2010) }\end{array}$ & Australia & Retrospective & Primary care & 372 & $76.1(7.8)$ & $\begin{array}{l}\text { Before } \\
\text { implementation of } \\
\text { the intervention }\end{array}$ & $7 / 9^{a}$ \\
\hline Lampela et al. (2010) & Finland & $\mathrm{RCT}$ & Primary care & $\begin{array}{l}\text { C: } 500 \\
\text { I: } 500\end{array}$ & $\geq 75$ & Standard care & $8 / 14^{b}$ \\
\hline Wessell et al. (2008) & United States & Prospective & Primary care & 124,802 & $\geq 65$ & - & $6 / 9^{a}$ \\
\hline $\begin{array}{l}\text { Spinewine et al. } \\
\text { (2007) }\end{array}$ & Belgium & $\mathrm{RCT}$ & Teaching hospital & $\begin{array}{l}\text { C: } 90 \\
\text { I: } 96\end{array}$ & $\begin{array}{l}\text { C: } 81.9(6.2) \\
\text { I: } 82.4(6.9)\end{array}$ & Usual care & $10 / 14^{b}$ \\
\hline
\end{tabular}


TABLE 1 | (Continued) Characteristics of the included studies $(n=47)$.

\begin{tabular}{|c|c|c|c|c|c|c|c|}
\hline \multirow[t]{2}{*}{ Author (year) } & \multirow[t]{2}{*}{ Country } & \multirow[t]{2}{*}{ Study design } & \multirow[t]{2}{*}{ Setting } & \multicolumn{2}{|c|}{ Elderly patients' sample } & \multirow[t]{2}{*}{ Comparator } & \multirow{2}{*}{$\begin{array}{c}\text { Quality } \\
\text { assessment/ } \\
\text { (score } \\
\text { obtained/total } \\
\text { score) }\end{array}$} \\
\hline & & & & Sample size & $\begin{array}{c}\text { Mean age } \\
\text { (SD or } \\
\text { IQR) }\end{array}$ & & \\
\hline $\begin{array}{l}\text { Brown and Earnhart } \\
\text { (2004) }\end{array}$ & United States & $\begin{array}{l}\text { Retrospective, case } \\
\text { series }\end{array}$ & Teaching hospital & 99 & 77.3 & - & $5 / 9^{a}$ \\
\hline Fick et al. (2004) & United States & $\mathrm{RCT}$ & Primary care & $\begin{array}{l}\text { C: } 185 \\
\text { I: } 170\end{array}$ & $\geq 65$ & Usual care & $6 / 14^{b}$ \\
\hline Allard et al. (2001) & Canada & $\mathrm{RCT}$ & Primary care & $\begin{array}{l}\text { C: } 130 \\
\text { I: } 136\end{array}$ & $\begin{array}{l}\text { C: } 80.7(4.6) \\
\text { I: } 80.4(4.3)\end{array}$ & Usual care & $10 / 14^{b}$ \\
\hline
\end{tabular}

A, after; B, before; $C$, control group; I, intervention group; IQR, interquartile range; $R C T$, randomized controlled trial; SD, standard deviation.

${ }^{a}$ The National Institutes of Health (NIH) quality assessment tool for case series studies.

${ }^{b}$ The National Institutes of Health (NIH) quality assessment tool of controlled intervention study.

${ }^{c}$ Median age.

TABLE 2 | Quality assessment of included studies through the National Institutes of Health (NIH) quality assessment tools.

\section{Quality assessment of controlled intervention studies}

\begin{tabular}{|c|c|c|c|c|}
\hline \multirow[t]{2}{*}{ No } & \multirow[t]{2}{*}{ Question } & \multicolumn{3}{|c|}{ Number of studies $(n=22)$} \\
\hline & & Yes & No & Other (CD, NA, NR) \\
\hline 1 & Was the study described as randomized, a randomized trial, a randomized clinical trial, or an RCT? & 16 & 6 & 0 \\
\hline 2 & Was the method of randomization adequate (i.e., use of randomly generated assignment)? & 11 & 2 & 9 \\
\hline 3 & Was the treatment allocation concealed (so that assignments could not be predicted)? & 10 & 3 & 9 \\
\hline 4 & Were study participants and providers blinded to treatment group assignment? & 6 & 11 & 5 \\
\hline 5 & Were the people assessing the outcomes blinded to the participants' group assignments? & 8 & 8 & 6 \\
\hline 6 & $\begin{array}{l}\text { Were the groups similar at baseline on important characteristics that could affect outcomes (e.g., demographics, risk } \\
\text { factors, co-morbid conditions)? }\end{array}$ & 19 & 2 & 1 \\
\hline 7 & Was the overall drop-out rate from the study at endpoint $20 \%$ or lower of the number allocated to treatment? & 13 & 9 & 0 \\
\hline 8 & Was the differential drop-out rate (between treatment groups) at endpoint 15 percentage points or lower? & 19 & 1 & 2 \\
\hline 9 & Was there high adherence to the intervention protocols for each treatment group? & 22 & 0 & 0 \\
\hline 10 & Were other interventions avoided or similar in the groups (e.g., similar background treatments)? & 21 & 1 & 0 \\
\hline 11 & Were outcomes assessed using valid and reliable measures, implemented consistently across all study participants? & 22 & 0 & 0 \\
\hline 12 & $\begin{array}{l}\text { Did the authors report that the sample size was sufficiently large to be able to detect a difference in the main outcome } \\
\text { between groups with at least } 80 \% \text { power? }\end{array}$ & 12 & 4 & 6 \\
\hline 13 & Were outcomes reported or subgroups analyzed prespecified (i.e., identified before analyses were conducted)? & 13 & 2 & 7 \\
\hline 14 & $\begin{array}{l}\text { Were all randomized participants analyzed in the group to which they were originally assigned, i.e., did they use an intention- } \\
\text { to-treat analysis? }\end{array}$ & 9 & 0 & 13 \\
\hline
\end{tabular}

\section{Quality assessment tool for case series studies}

No

Question

Number of Studies $(n=25)$

Was the study question or objective clearly stated?

Was the study population clearly and fully described, including a case definition?

Were the cases consecutive?

Were the subjects comparable?

Was the intervention clearly described?

Were the outcome measures clearly defined, valid, reliable, and implemented consistently across all study participants?

Was the length of follow-up adequate?

Were the statistical methods described well?

Were the results described well?

$\begin{array}{ccc}\text { Yes } & \text { No } & \text { Other (CD, NA, NR) } \\ 25 & 0 & 0 \\ 24 & 0 & 1 \\ 4 & 6 & 15 \\ 25 & 0 & 0 \\ 22 & 3 & 0 \\ 22 & 3 & 0 \\ 4 & 0 & 21 \\ 21 & 4 & 0 \\ 24 & 1 & 0\end{array}$

Earnhart, 2004; Fick et al., 2004; Wessell et al., 2008; Castelino et al., 2010; Lampela et al., 2010; Franchi et al., 2014; Franchi et al., 2016; Moss et al., 2016, 2019; Stevens et al., 2017; Vanderman et al., 2017; Chan et al., 2018; Stuckey et al., 2018; Vandenberg et al., 2018; Liu et al., 2019; Vu and Huong, 2019), Screening Tool of Older People's potentially inappropriate Prescriptions 
TABLE 3 | Effects of medication review interventions on inappropriate prescribing in older adults $(n=23)$.

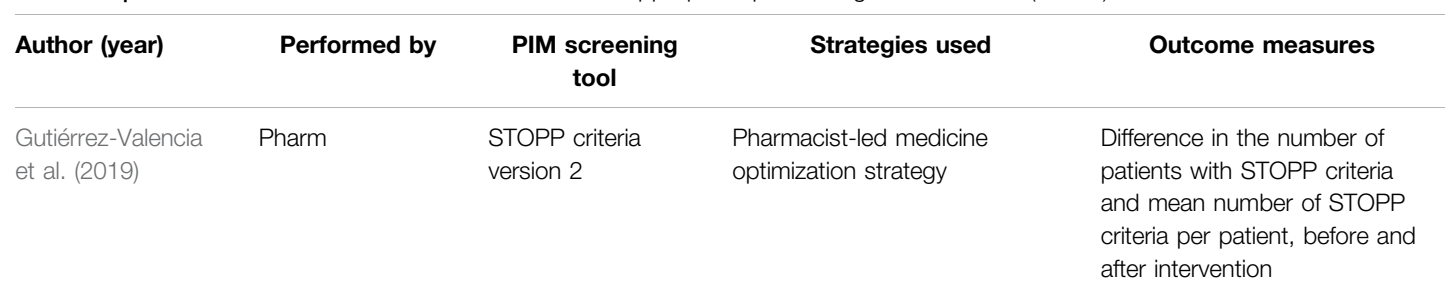

$\begin{array}{llll}\text { Khera et al. (2019) Pharm } & \begin{array}{l}2015 \text { Beers and } \\ \text { version } 2 \text { of STOPP } \\ \text { criteria }\end{array} & \begin{array}{l}\text { Pharmacist-led medication } \\ \text { review }\end{array} & \begin{array}{l}\text { Number of medications } \\ \text { satisfying explicit criteria of } \\ \text { STOPP/Beers for PIM }\end{array}\end{array}$

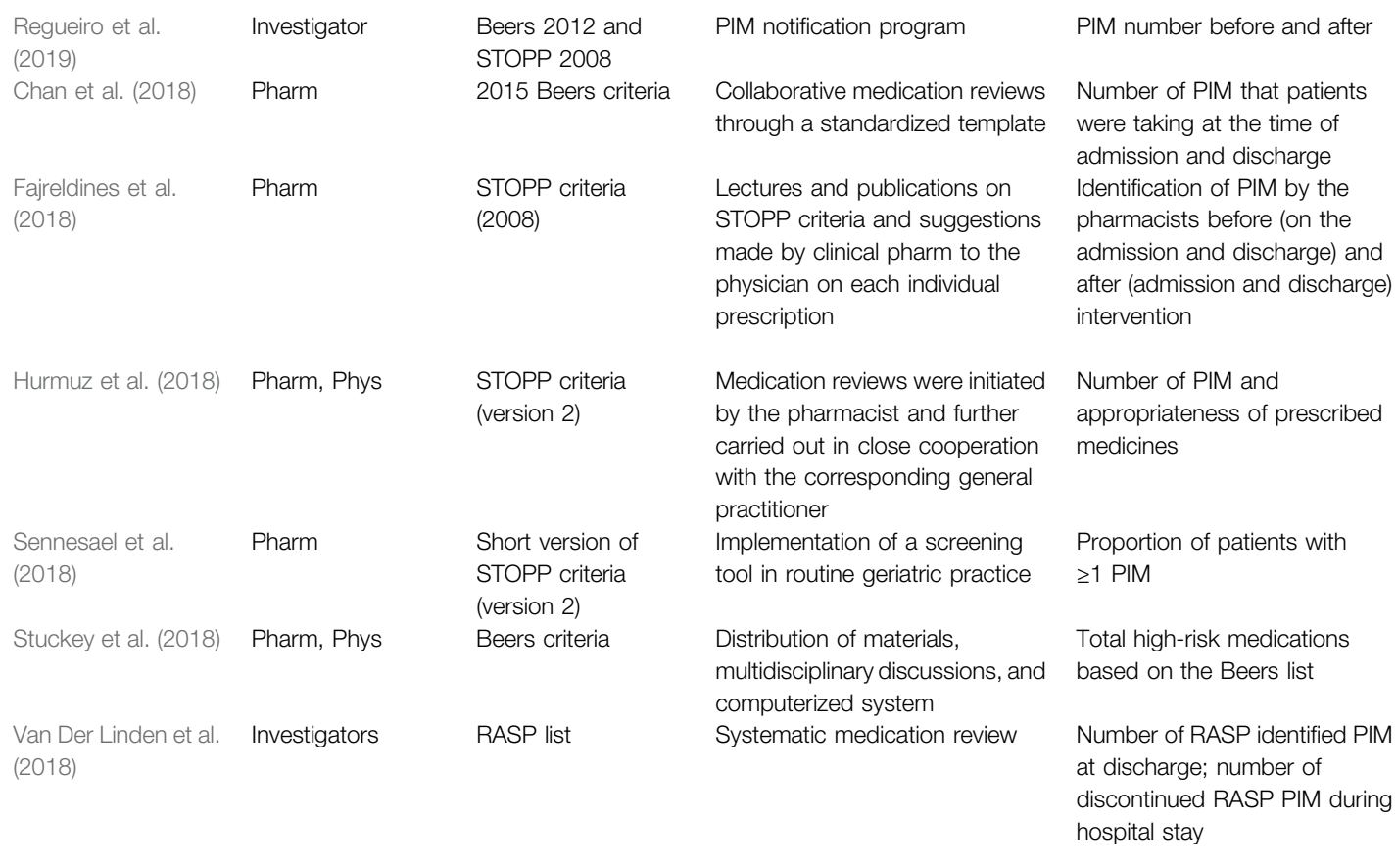

Frankenthal et al. Study pharm (2017)

Van der Linden et al. Pharm (2017)

Campins et al. Pharm version 2 of STOPP review

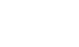


TABLE 3 | (Continued) Effects of medication review interventions on inappropriate prescribing in older adults ( $\mathrm{n}=23)$.

\begin{tabular}{|c|c|c|c|c|c|}
\hline Author (year) & Performed by & $\begin{array}{c}\text { PIM screening } \\
\text { tool }\end{array}$ & Strategies used & Outcome measures & Significant outcomes \\
\hline & & & $\begin{array}{l}\text { patient. A final decision was } \\
\text { agreed by physicians and their } \\
\text { patients in a face-to-face visit }\end{array}$ & & \\
\hline Tallon et al. (2015) & Pharm & MAI & $\begin{array}{l}\text { Collaborative PACT model on the } \\
\text { medication appropriateness of } \\
\text { acute hospitalized older patients }\end{array}$ & $\begin{array}{l}\text { Appropriateness of prescribing } \\
\text { at pre-admission, during } \\
\text { admission, and at discharge }\end{array}$ & $\begin{array}{l}\text { PACT significantly improved } \\
\text { the MAI score from pre- } \\
\text { admission to admission (mean } \\
\text { difference } 2.4,95 \% \mathrm{Cl} 1.0 \text { to } \\
3.9, p<0.005 \text { ) and from pre- } \\
\text { admission to discharge (mean } \\
\text { difference } 4.0,95 \mathrm{Cl} 1.7 \text { to } 6.4 \text {, } \\
p<0.005 \text { ) } \\
\text { PACT resulted in significantly } \\
\text { fewer drugs with } 1 \text { or more } \\
\text { inappropriate rating at } \\
\text { discharge (PACT } 15.0 \% \text {, } \\
\text { standard } 30.5 \%, p<0.001 \text { ) }\end{array}$ \\
\hline Dalleur et al. (2014) & Ger & $\begin{array}{l}\text { STOPP criteria } \\
(2008)\end{array}$ & $\begin{array}{l}\text { STOPP criteria } \\
\text { recommendations from an } \\
\text { inpatient geriatric consultation } \\
\text { team }(\mathrm{GCT})\end{array}$ & Proportion of PIM discontinued & $\begin{array}{l}\text { Discontinuation at discharge of } \\
\text { PIM present on admission } \\
\text { C: } 19.3 \% \text { vs. I: } 39.7 \% \\
p=0.013\end{array}$ \\
\hline $\begin{array}{l}\text { Frankenthal et al. } \\
\text { (2014) }\end{array}$ & Study pharm & $\begin{array}{l}\text { STOPP criteria } \\
(2008)\end{array}$ & $\begin{array}{l}\text { Medication review for all } \\
\text { residents at study opening and } 6 \\
\text { and } 12 \text { months later based on } \\
\text { STOPP criteria }\end{array}$ & Number of PIM over time & $\begin{array}{l}\text { Number of PIM at baseline } \\
\text { C: } 114(64.7 \%) \text { vs. I: } \\
129(70.5 \%) \\
\text { Number of PIM after 6-month } \\
\text { follow-up } \\
\text { C: } 89(56 \%) \text { vs. I: } 65(37.4 \%) \text {, } \\
p=0.001 \\
\text { Number of PIM after } 12 \text {-month } \\
\text { follow up } \\
\text { C: } 79(54.1 \%) \text { vs. I: } 36(22.5 \%) \text {, } \\
p<0.001\end{array}$ \\
\hline Lopatto et al. (2014) & Phys & Maio criteria & $\begin{array}{l}\text { Participatory clinical guidelines } \\
\text { development, group educational } \\
\text { outreach, and dissemination of } \\
\text { educational materials combined } \\
\text { with peer-to-peer interactive } \\
\text { discussion }\end{array}$ & PIM incidence rate & Not achieved \\
\hline Keith et al. (2013) & Phys & Maio criteria & $\begin{array}{l}\text { Participatory clinical guidelines } \\
\text { development, group educational } \\
\text { outreach, and dissemination of } \\
\text { educational materials combined } \\
\text { with peer-to-peer interactive } \\
\text { discussion }\end{array}$ & $\begin{array}{l}\text { Quarterly incidence rates of } \\
\text { older patients exposed to PIM }\end{array}$ & $\begin{array}{l}\text { Patients exposed to at least } \\
1 \text { PIM } \\
2007 \\
\text { C: } 6,315(7.7 \%) \text { vs. I: } \\
6,098(7.7 \%) \\
2009 \\
\text { C: } 5,111(6.1 \%) \text { vs. I: } 4,277 \\
(5.3 \%) p<0.001\end{array}$ \\
\hline $\begin{array}{l}\text { Gallagher et al. } \\
\text { (2011) }\end{array}$ & Phys & 2008 STOPP criteria & $\begin{array}{l}\text { STOPP screening and } \\
\text { recommendations to the } \\
\text { attending medical team }\end{array}$ & $\begin{array}{l}\text { Patients with } \geq 1 \text { STOPP criteria } \\
\text { at discharge }\end{array}$ & $\begin{array}{l}\text { Patients with } \geq 1 \text { STOPP } \\
\text { criteria at discharge } \\
\text { C: } 93(48.4 \%) ; \text { I: } 7(3.7 \%), \\
p<0.001\end{array}$ \\
\hline $\begin{array}{l}\text { Castelino et al. } \\
(2010)\end{array}$ & Pharm & 2003 Beers criteria & $\begin{array}{l}\text { Home Medicine Review (HMR) } \\
\text { service }\end{array}$ & Rate of PIM & Not achieved \\
\hline Lampela et al. (2010) & $\begin{array}{l}\text { Phys, N, } \\
\text { physiotherapist, } \\
\text { nutritionist }\end{array}$ & $\begin{array}{l}1997 \text { Beers criteria } \\
\text { (US } 2003 \text { update) }\end{array}$ & $\begin{array}{l}\text { Adjustment of a patient's } \\
\text { medication when necessary; } \\
\text { evaluation of the indications for all } \\
\text { drugs in use; clinical examination, } \\
\text { including careful evaluation of } \\
\text { cognition, mood, orthostatic } \\
\text { reactions, and presence of } \\
\text { extrapyramidal symptoms; } \\
\text { routine blood tests }\end{array}$ & $\begin{array}{l}\text { Numbers of inappropriate } \\
\text { drugs or dosages }\end{array}$ & Not achieved \\
\hline $\begin{array}{l}\text { Spinewine et al. } \\
\text { (2007) }\end{array}$ & Pharm & $\begin{array}{l}\text { MAI, Beers (1997), } \\
\text { and ACOVE criteria }\end{array}$ & $\begin{array}{l}\text { The appropriateness of } \\
\text { treatment was analyzed, and a } \\
\text { pharmaceutical care plan was }\end{array}$ & $\begin{array}{l}\text { Appropriateness of prescribing } \\
\text { at admission, discharge, and }\end{array}$ & $\begin{array}{l}\text { Intervention patients } \\
\text { significantly more likely than } \\
\text { control patients to have } \\
\text { (Continued on following page) }\end{array}$ \\
\hline
\end{tabular}


TABLE 3 | (Continued) Effects of medication review interventions on inappropriate prescribing in older adults ( $\mathrm{n}=23$ ).

\begin{tabular}{|c|c|c|c|c|c|}
\hline Author (year) & Performed by & $\begin{array}{l}\text { PIM screening } \\
\text { tool }\end{array}$ & Strategies used & Outcome measures & Significant outcomes \\
\hline & & & $\begin{array}{l}\text { prepared. Whenever an } \\
\text { opportunity for optimization was } \\
\text { identified, the pharmacist } \\
\text { discussed that opportunity with } \\
\text { the prescriber, who could accept } \\
\text { or reject the intervention }\end{array}$ & $\begin{array}{l}3 \text { months after discharge using } \\
\text { Beers' criteria }\end{array}$ & $\begin{array}{l}\text { improvements in Beers' criteria } \\
\text { [OR } 0.6(95 \% \mathrm{Cl} 0.3,1.1)]\end{array}$ \\
\hline $\begin{array}{l}\text { Brown and Earnhart } \\
\text { (2004) }\end{array}$ & Pharm & Beers criteria (1997) & $\begin{array}{l}\text { Acute Care for Elders (ACE) team } \\
\text { improvement on the medication } \\
\text { regime of geriatric inpatients }\end{array}$ & Prevalence of PIM & $\begin{array}{l}\text { Rate of PIM at admission } \\
10.1 \% \text {, and discharge } 2.02 \% \text {, } \\
p<0.02\end{array}$ \\
\hline Allard et al. (2001) & Pharm, N, Phys & $\begin{array}{l}\text { List of PIM } \\
\text { developed by the } \\
\text { Quebec Committee } \\
\text { on Drug Use in the } \\
\text { Elderly }\end{array}$ & $\begin{array}{l}\text { A team comprising } 2 \text { physicians, } \\
\text { a pharmacist, and a nurse } \\
\text { reviewed the list of drugs and the } \\
\text { diagnoses of patients and } \\
\text { formulated suggestions that } \\
\text { were mailed to the patients' } \\
\text { physician }\end{array}$ & $\begin{array}{l}\text { Number of PIM; number of } \\
\text { subjects with at least } 1 \text { PIM }\end{array}$ & Not achieved \\
\hline
\end{tabular}

A, after group; B, before group; C, control group; Cl, confidence interval; Ger, geriatrician; I, intervention group; IQR, interquartile range; MAl, Medication Appropriateness Index; N, nurse; Pharm, pharmacist; Phys, physician; PACT, pharmaceutical care at Tallaght Hospital; PIM, potentially inappropriate medication; RASP, Rationalization of Home Medication by an Adjusted STOPP list in Older Patient; SD, standard deviation; STOPP, Screening Tool of Older People's potentially inappropriate Prescriptions; US, United States.

(STOPP) criteria $(n=15)$ (Gallagher et al., 2011; Dalleur et al., 2014; Frankenthal et al., 2014; Campins et al., 2016; Urfer et al., 2016; Frankenthal et al., 2017; Price et al., 2017; Fajreldines et al., 2018; Gibert et al., 2018; Hurmuz et al., 2018; Sennesael et al., 2018; Boersma et al., 2019; Gutiérrez-Valencia et al., 2019; Akkawi et al., 2020; Winata et al., 2020), a combination of Beers and STOPP criteria $(n=5)$ (Ilić et al., 2015; Etxeberria et al., 2018; Najjar et al., 2018; Khera et al., 2019; Regueiro et al., 2019), and Medication Appropriateness Index (MAI) $(n=1)$ (Tallon et al., 2015). Five studies used self-developed or adapted criteria (Allard et al., 2001; Keith et al., 2013; Lopatto et al., 2014; Van der Linden et al., 2017; Van Der Linden et al., 2018), and the remaining studies used a combination of validated (STOPP and/or Beers) and self-developed or adapted criteria.

\subsubsection{Interventions Used}

After thorough analysis of the studies, five different types of interventions have emerged: medication review, educational interventions, CDSS, multifaceted approaches, and organizational strategies (Tables 3-7, respectively). Twentythree studies used a medication review approach (Allard et al., 2001; Brown and Earnhart, 2004; Spinewine et al., 2007; Castelino et al., 2010; Lampela et al., 2010; Gallagher et al., 2011; Keith et al., 2013; Dalleur et al., 2014; Frankenthal et al., 2014; Lopatto et al., 2014; Tallon et al., 2015; Campins et al., 2016; Frankenthal et al., 2017; Van der Linden et al., 2017; Chan et al., 2018; Fajreldines et al., 2018; Hurmuz et al., 2018; Sennesael et al., 2018; Stuckey et al., 2018; Van Der Linden et al., 2018; Gutiérrez-Valencia et al., 2019; Khera et al., 2019; Regueiro et al., 2019), an educational intervention was the strategy used in eight studies (Fick et al., 2004; Rognstad et al., 2013; Franchi et al., 2014, 2016; Ilić et al., 2015; Clyne et al., 2016; Etxeberria et al., 2018; Moss et al., 2019), multifaceted approach was present in nine (Clyne et al., 2016; Moss et al., 2016; Gibert et al., 2018; Najjar et al., 2018;
Vandenberg et al., 2018; Boersma et al., 2019; Liu et al., 2019; Vu and Huong, 2019; Akkawi et al., 2020) (i.e., a combination of different interventions), five studies used a CDSS (Urfer et al., 2016; Price et al., 2017; Vanderman et al., 2017; McDonald et al., 2019; Winata et al., 2020), and two used organizational strategies (Wessell et al., 2008; Stevens et al., 2017) (i.e., regulatory policies developed to decrease the number of PIM).

\subsubsection{Impact of Medication Review Interventions}

Medication review was conducted by different healthcare professionals: physicians (Gallagher et al., 2011; Keith et al., 2013; Lopatto et al., 2014), pharmacists (Brown and Earnhart, 2004; Spinewine et al., 2007; Castelino et al., 2010; Frankenthal et al., 2014; Tallon et al., 2015; Campins et al., 2016; Van der Linden et al., 2017; Chan et al., 2018; Fajreldines et al., 2018; Sennesael et al., 2018; Gutiérrez-Valencia et al., 2019; Khera et al., 2019), pharmacists and physicians (Hurmuz et al., 2018; Stuckey et al., 2018), gerontologists (Dalleur et al., 2014), investigators (Van Der Linden et al., 2018; Regueiro et al., 2019), pharmacist students (Frankenthal et al., 2017), and multidisciplinary teams (Allard et al., 2001; Lampela et al., 2010). Although medication review intervention varies in the included studies, this intervention involved the analysis of the patient's pharmacotherapeutic needs and prescribed drugs, followed by a recommendation to optimize medication.

Six (Brown and Earnhart, 2004; Spinewine et al., 2007; Tallon et al., 2015; Van der Linden et al., 2017; Fajreldines et al., 2018; Gutiérrez-Valencia et al., 2019) of eight medication review interventions conducted by pharmacists at the hospital (Brown and Earnhart, 2004; Spinewine et al., 2007; Tallon et al., 2015; Van der Linden et al., 2017; Chan et al., 2018; Fajreldines et al., 2018; Sennesael et al., 2018; Gutiérrez-Valencia et al., 2019) demonstrate a positive impact on PIM reduction. Among these, in two studies the intervention improved the PIM 
TABLE 4 | Effects of educational interventions on inappropriate prescribing in older adults $(n=8)$.

\begin{tabular}{|c|c|c|c|c|c|c|}
\hline $\begin{array}{l}\text { Author } \\
\text { (year) }\end{array}$ & Performed by & Receivers & $\begin{array}{c}\text { PIM screening } \\
\text { tool }\end{array}$ & Strategies used & Outcome measures & Significant outcomes \\
\hline $\begin{array}{l}\text { Moss et al. } \\
(2016)\end{array}$ & Pharm, Phys & $\begin{array}{l}\text { Medical } \\
\text { residents }\end{array}$ & $\begin{array}{l}\text { Table } 2 \text { of } 2012 \text { Beers } \\
\text { criteria }\end{array}$ & $\begin{array}{l}\text { Enhancing Quality of } \\
\text { Prescribing Practices for } \\
\text { Veterans Discharged from the } \\
\text { Emergency Department } \\
\text { (EQUiPPED) provider } \\
\text { education through academic } \\
\text { detailing, clinical decision } \\
\text { support, and provider } \\
\text { feedback on prescribing } \\
\text { practices }\end{array}$ & $\begin{array}{l}\text { Prescription rate ratio } \\
\text { before and after the } \\
\text { intervention }\end{array}$ & $\begin{array}{l}\text { The group after the intervention } \\
\text { were less likely to prescribe a } \\
\text { PIM when compared to the } \\
\text { group before the intervention } \\
\text { (rate ratio }=0.73,95 \% \\
\mathrm{Cl}=0.632-0.850 ; p<0.0001 \text { ) }\end{array}$ \\
\hline $\begin{array}{l}\text { Etxeberria } \\
\text { et al. (2018) }\end{array}$ & $\begin{array}{l}\text { Research } \\
\text { team }\end{array}$ & Phys & STOPP and Beers criteria & $\begin{array}{l}\text { Electronic identification of } \\
\text { PIM, training for physicians } \\
\text { and structured review of } \\
\text { medication }\end{array}$ & $\begin{array}{l}\text { Change in the number } \\
\text { of PIM per patient }\end{array}$ & $\begin{array}{l}\text { Number of PIM/patients (SD) } \\
\text { B: } 0.70(0.91) \text { vs. A: } 0.51(0.77) \text {, } \\
p<0.0001\end{array}$ \\
\hline $\begin{array}{l}\text { Clyne et al. } \\
\text { (2016) }\end{array}$ & Pharm & $\begin{array}{l}\text { Phys (C: 10, } \\
\text { I: 11) }\end{array}$ & $\begin{array}{l}\text { Beers, STOPP, McLeod, } \\
\text { IPET, ACOVE, and the } \\
\text { Prescription Peer Academic }\end{array}$ & $\begin{array}{l}\text { Academic detailing, review of } \\
\text { medicines with web-based } \\
\text { pharmaceutical treatment }\end{array}$ & $\begin{array}{l}\text { Proportion of patients } \\
\text { with PIM and mean } \\
\text { number of PIM }\end{array}$ & $\begin{array}{l}\text { Mean number of PIM (SD) } \\
\text { C: } 1.03(0.8) \text { vs. I: } 0.61(0.7) \\
p=0.01\end{array}$ \\
\hline
\end{tabular}

\begin{tabular}{|c|c|c|c|c|}
\hline $\begin{array}{l}\text { Franchi et al. } \\
\text { (2016) }\end{array}$ & $\begin{array}{l}\text { Research } \\
\text { team }\end{array}$ & Phys & 2012 Beers criteria & $\begin{array}{l}\text { E-learning educational } \\
\text { program }\end{array}$ \\
\hline
\end{tabular}

\begin{tabular}{|c|c|c|c|c|}
\hline $\begin{array}{l}\text { Ilić et al. } \\
(2015)\end{array}$ & $\begin{array}{l}\text { Investigator } \\
\text { (Phys) }\end{array}$ & 27 Phys & $\begin{array}{l}2012 \text { Beers criteria and } \\
2008 \text { STOPP criteria }\end{array}$ & Lectures and brochures \\
\hline
\end{tabular}

\begin{tabular}{|c|c|c|c|c|c|c|}
\hline $\begin{array}{l}\text { Franchi et al. } \\
\text { (2014) }\end{array}$ & $\begin{array}{l}\text { Research } \\
\text { Team }\end{array}$ & $\begin{array}{l}\text { Phys (C: } 22 \text {, } \\
\text { I: } 54 \text { ) }\end{array}$ & 2012 Beers criteria & $\begin{array}{l}\text { E-learning educational } \\
\text { program }\end{array}$ & $\begin{array}{l}\text { Reduction of } \\
\text { prescription of PIM }\end{array}$ & $\begin{array}{l}(0.0-6.0), p<0.005 \\
\text { Not achieved }\end{array}$ \\
\hline $\begin{array}{l}\text { Rognstad } \\
\text { et al. (2013) }\end{array}$ & $\begin{array}{l}\text { Phys (peer } \\
\text { academic } \\
\text { detailers) }\end{array}$ & $\begin{array}{l}\text { Phys (C: } \\
\text { 209, I: 256) }\end{array}$ & $\begin{array}{l}13 \text { explicit PIM criteria, } \\
\text { assumed to be relevant for } \\
\text { the Norwegian general } \\
\text { practice setting (based on } \\
\text { Beers criteria and The } \\
\text { Swedish National Board of } \\
\text { Health and Welfare) }\end{array}$ & $\begin{array}{l}\text { Multifaceted educational } \\
\text { intervention with feedback } \\
\text { and audit }\end{array}$ & $\begin{array}{l}\text { Changes in } \\
\text { prescription patterns }\end{array}$ & Not achieved \\
\hline $\begin{array}{l}\text { Fick et al. } \\
(2004)\end{array}$ & $\begin{array}{l}\text { Research } \\
\text { team }\end{array}$ & $\begin{array}{l}\text { Phys (C: } \\
\text { 185, l: 170) }\end{array}$ & $\begin{array}{l}1997 \text { Beers criteria for } \\
\text { medications to avoid in } \\
\text { older adults }\end{array}$ & $\begin{array}{l}\text { Integrated decision support } \\
\text { service: 1) a detailed } \\
\text { educational brochure listing } \\
\text { PIM, 2) a list of suggested PIM } \\
\text { alternative medications, and } \\
\text { 3) a personally addressed } \\
\text { letter that described in detail } \\
\text { all the physician's patients } \\
\text { who were determined to be in } \\
\text { receipt of } 1 \text { or more PIM }\end{array}$ & $\begin{array}{l}\text { Rate of providers that } \\
\text { prescribed at least } \\
1 \mathrm{PIM}\end{array}$ & $\begin{array}{l}\text { Number of continuously enrolled } \\
\text { members with at least } 1 \text { PIM } \\
\text { declined significantly } \\
(\chi 2=13.20, p<0.001) \text { to } \\
17.9 \%(3,007 / 16,818) \text {, from a } \\
\text { baseline of } 19.4 \%(3,364 / \\
17,330)\end{array}$ \\
\hline
\end{tabular}

A, after group; B, before group; C, control group; I, intervention group; Pharm, pharmacist; Phys, physician; PIM, potentially inappropriate medication; SD, standard deviation; STOPP, Screening Tool of Older People's potentially inappropriate Prescriptions.

screening tool score (Spinewine et al., 2007; Tallon et al., 2015). Fajreldines et al. (2018) and Gutiérrez-Valencia et al. (2019) reported that the number of patients with PIM significantly decreased after medication review $(19.2 \%$ and $30.7 \%$, respectively). Van der Linden et al. (2017) observed a decrease in the average number of PIM after an intervention. Finally, Brown and Earnhart (2004) conclude that their intervention led to an $8.08 \%$ absolute risk reduction.

Among the studies performed by physicians in hospitals, one was conducted at an emergency department of a hospital (Gallagher 
TABLE 5 | Effects of clinical decision support system (CDSS) interventions on inappropriate prescribing in older adults $(n=5)$.

\begin{tabular}{|c|c|c|c|c|}
\hline Author (year) & PIM screening tool & Strategies used & Outcome measures & Significant outcomes \\
\hline $\begin{array}{l}\text { Winata et al. } \\
(2020)\end{array}$ & STOPP version 2 & $\begin{array}{l}\text { Introduction of an electronic medication } \\
\text { management system (EMMS) }\end{array}$ & $\begin{array}{l}\text { Number of PIM on admission and discharge } \\
\text { per patient; number of patients with } \geq 1 \text { PIM } \\
\text { on admission and discharge }\end{array}$ & Not achieved \\
\hline $\begin{array}{l}\text { McDonald et al. } \\
\text { (2019) }\end{array}$ & $\begin{array}{l}\text { Beers and STOPP criteria } \\
\text { (version 2), and Choosing } \\
\text { Wisely lists }\end{array}$ & $\begin{array}{l}\text { Electronic decision support tool that } \\
\text { generates deprescribing opportunities } \\
\text { reports }\end{array}$ & $\begin{array}{l}\text { Proportion of patients with } 1 \text { or more home } \\
\text { medications identified as a PIM and } \\
\text { deprescribed at hospital discharge }\end{array}$ & Not achieved \\
\hline $\begin{array}{l}\text { Price et al. } \\
(2017)\end{array}$ & STOPP criteria & $\begin{array}{l}\text { Electronic medical record with automated } \\
\text { STOPP rules }\end{array}$ & $\begin{array}{l}\text { Change in measured PIM rates between the } \\
\text { intervention and control groups before the } \\
\text { intervention as compared with the difference } \\
\text { after the intervention period }\end{array}$ & Not achieved \\
\hline $\begin{array}{l}\text { Vanderman } \\
\text { et al. (2017) }\end{array}$ & 2012 Beers criteria & Medication alert message & Overall PIM, top 10 PIM, and flagged PIM & $\begin{array}{l}\text { New top } 10 \mathrm{PIM} / \text { new total } \\
\text { medications } \\
\text { B: } 1,405 / 15,539(12.56 \%) \\
\text { vs. A: } 1,308 / 15,807 \\
(12.00 \%), p=0.0158\end{array}$ \\
\hline $\begin{array}{l}\text { Urfer et al. } \\
(2016)\end{array}$ & 2008 STOPP criteria & $\begin{array}{l}\text { Easy-to-use 5-point checklist: 1) ascertain all } \\
\text { current medications used; 2) identify patients } \\
\text { at high risk of adverse drug reactions; 3) } \\
\text { estimate life expectancy; 4) identify } \\
\text { medications which are not indicated and/or } \\
\text { are potentially dangerous; and 5) monitor the } \\
\text { patient if drugs were stopped or new drugs } \\
\text { were added }\end{array}$ & $\begin{array}{l}\text { Proportion of patients prescribed PIM at } \\
\text { discharge }\end{array}$ & $\begin{array}{l}\text { Patients with }>1 \text { PIM at } \\
\text { discharge } \\
\text { B: } 164(39.0 \%) \text { vs. A: } 102 \\
(23.7 \%), p<0.001\end{array}$ \\
\hline
\end{tabular}

A, after group; B, before group; PIM, potentially inappropriate medication; STOPP, Screening Tool of Older People's potentially inappropriate Prescriptions.

et al., 2011), and the remaining two studies used the local health authority databases (Keith et al., 2013; Lopatto et al., 2014). Gallagher et al. (2011) observed a significant reduction in the proportion of patients with at least one PIM in the intervention group (from $43.2 \%$ in admission to $3.7 \%$, at discharge). This trend remains stable during the 6 months of follow-up. One study performed a quality improvement program across an Italian region with more than 80,000 older adults and observed that the PIM exposure incidence rate significantly declined $31.4 \%$ (from the baseline to the postintervention period) (Keith et al., 2013).

The intervention performed by a gerontologist in hospitalized patients results in a significant decrease in the number of PIM in patients discharged (PIM discontinuation of 39.7\%) (Dalleur et al., 2014). Similar outcomes were achieved in the intervention performed by investigators in hospitalized patients (Van Der Linden et al., 2018).

In primary care, the medication review performed by both physicians and pharmacists results in a significant reduction in the mean number of PIM per patient [from 0.6 to 0.4 (Hurmuz et al., 2018) and from 1.24 to 0.82 (Stuckey et al., 2018)]. In this setting, a multidisciplinary team failed to achieve a significant reduction in the number of PIM, and only one (Khera et al., 2019) of the three pharmacists' interventions (Castelino et al., 2010; Campins et al., 2016; Khera et al., 2019) results in a significant impact in PIM reduction.

Finally, in a chronic care geriatric facility, pharmacy students observed a decline in PIM prescriptions after a follow-up of 12 and 24 months (Frankenthal et al., 2014; Frankenthal et al., 2017).

\subsubsection{Impact of Educational Interventions}

In the eight included studies (Fick et al., 2004; Rognstad et al., 2013; Franchi et al., 2014, 2016; Ilić et al., 2015; Clyne et al., 2016;
Etxeberria et al., 2018; Moss et al., 2019) that used educational approaches to reduce PIM, the interventions were performed by the following: a researcher and/or a research team (Fick et al., 2004; Franchi et al., 2014, 2016; Ilić et al., 2015; Etxeberria et al., 2018), a multifaceted team containing a pharmacist and a physician (Moss et al., 2019) or physicians (peer academic detailers) (Rognstad et al., 2013), or a pharmacist (Clyne et al., 2016). In all studies the target of the educational interventions were physicians. The outcomes of the interventions were measured through the reduction of PIM use or PIM prescriptions. In one of the studies, the included population was polymedicated older adults (Etxeberria et al., 2018); in the three studies that have a positive impact on PIM, the average number of PIM per patient ranged from $0.7-11$ before intervention to $0.51-1.5$ after the intervention (Ilić et al., 2015; Clyne et al., 2016; Etxeberria et al., 2018). One study reported that the number of physicians that prescribed at least one PIM declined $17.9 \%$ (Fick et al., 2004). Finally, one study reported that after the educational intervention the PIM rate ratio before and after the intervention is 0.73 (Moss et al., 2019).

\subsubsection{Impact of Clinical Decision Support System Interventions}

Five studies used CDSS to reduce PIM (Urfer et al., 2016; Price et al., 2017; Vanderman et al., 2017; McDonald et al., 2019; Winata et al., 2020). Two of four studies performed in hospitalized patients reported that the implementation of CDSS has a positive impact on PIM deprescription (Urfer et al., 2016; Vanderman et al., 2017). In one study, the introduction of a PIM checklist, in an internal medicine ward, leads to a significant reduction $(22.0 \%)$ of the risk of 
TABLE 6 | Effects of multifaceted interventions on inappropriate prescribing in older adults $(n=9)$.

\begin{tabular}{|c|c|c|c|c|c|c|c|c|}
\hline \multirow{2}{*}{$\begin{array}{l}\text { Author } \\
\text { (year) }\end{array}$} & \multicolumn{2}{|c|}{ Educational } & \multirow[t]{2}{*}{ CDSS } & \multirow{2}{*}{$\begin{array}{l}\text { Medication } \\
\text { review }\end{array}$} & \multirow{2}{*}{$\begin{array}{c}\text { PIM screening } \\
\text { tool }\end{array}$} & \multirow[t]{2}{*}{ Strategies used } & \multirow[t]{2}{*}{ Outcome measures } & \multirow{2}{*}{$\begin{array}{l}\text { Significant } \\
\text { outcomes }\end{array}$} \\
\hline & Performed by & Receivers & & & & & & \\
\hline $\begin{array}{l}\text { Akkawi et al. } \\
\text { (2020) }\end{array}$ & Not reported & Phys, Pharm & $x$ & & $\begin{array}{l}\text { STOPP } \\
\text { version } 2\end{array}$ & $\begin{array}{l}\text { Three educational } \\
\text { sessions about PIM } \\
\text { and discussion of } \\
\text { STOPP criteria, } \\
\text { coupled with an } \\
\text { introduction of a } \\
\text { CDSS }\end{array}$ & PIM prevalence & Not achieved \\
\hline $\begin{array}{l}\text { Boersma } \\
\text { et al. (2019) }\end{array}$ & & & $x$ & Phys & $\begin{array}{l}\text { STOPP } \\
\text { version } 1\end{array}$ & $\begin{array}{l}\text { The intervention } \\
\text { consisted of written } \\
\text { prescribing } \\
\text { recommendations } \\
\text { prepared by an } \\
\text { independent, } \\
\text { clinically experienced } \\
\text { research physician } \\
\text { using the STRIP } \\
\text { Assistant }\end{array}$ & $\begin{array}{l}\text { PIM changes } \\
\text { implementation }\end{array}$ & $\begin{array}{l}\text { PIM changes } \\
\text { implementations } \\
\text { C: } 15.3 \% \text { vs. I: } 46.2 \% \\
\text { ( } p<0.001 \text { ) }\end{array}$ \\
\hline $\begin{array}{l}\text { Liu et al. } \\
\text { (2019) }\end{array}$ & & & $x$ & Pharm, Phys & $\begin{array}{l}\text { Modified and } \\
\text { updated } 2015 \\
\text { Beers criteria } \\
\text { according to } \\
\text { common } \\
\text { practice and } \\
\text { culture in } \\
\text { Taiwan }\end{array}$ & $\begin{array}{l}\text { Creation of a } \\
\text { multidisciplinary Chi- } \\
\text { Mei Integrated } \\
\text { Geriatric Emergency } \\
\text { Team; creation of a } \\
\text { PIM list; computer- } \\
\text { based medication } \\
\text { reconciliation and } \\
\text { integration system to } \\
\text { obtain information } \\
\text { about medications } \\
\text { prescribed }\end{array}$ & $\begin{array}{l}\text { Number of PIM at } \\
\text { hospital admission and } \\
\text { discharge }\end{array}$ & $\begin{array}{l}\text { Number of PIM on } \\
\text { admission } \\
\text { B: } 173 \text { vs. A: } 480 \text {, } \\
\text { and at discharge } \\
\text { B: } 88 \text { vs. A: } 156 . \\
p<0.001\end{array}$ \\
\hline $\begin{array}{l}\text { Vu and } \\
\text { Huong } \\
\text { (2019) }\end{array}$ & Pharm & Phys & & Pharm & $\begin{array}{l}2015 \text { Beers } \\
\text { criteria }\end{array}$ & $\begin{array}{l}\text { Pharmacists gave a } \\
\text { training lecture on the } \\
\text { Beers } 2015 \text { criteria in } \\
2 \mathrm{~h} \text { for the medical } \\
\text { doctors; the training } \\
\text { was also conducted } \\
\text { as face-to-face visits. } \\
\text { The notebook with } \\
\text { the Beers } 2015 \\
\text { criteria was provided } \\
\text { to the medical } \\
\text { doctors }\end{array}$ & Prevalence of PIM & $\begin{array}{l}\text { Prevalence of PIM } \\
\text { B: } 34.1 \% \text { vs. A: } \\
23.1 \% \text {, (odds ratio } \\
(\mathrm{OR})=0.337,95 \% \\
\mathrm{Cl}=0.207-0.551 \\
p<0.001)\end{array}$ \\
\hline $\begin{array}{l}\text { Najjar et al. } \\
(2018)\end{array}$ & $\begin{array}{l}\text { Head of geriatric } \\
\text { medicine, } 2 \\
\text { Pharms }\end{array}$ & Pharm & & Pharm, Phys & $\begin{array}{l}\text { STOPP (version } \\
\text { 2) and Beers } \\
\text { (2015) criteria }\end{array}$ & $\begin{array}{l}\text { Educational program } \\
\text { consisting of 1-h, } \\
\text { weekly educational } \\
\text { lectures for } 1 \text { month, } \\
\text { handbook designed } \\
\text { was distributed to } \\
\text { the physicians at the } \\
\text { end of the seminars; } \\
\text { collaboration } \\
\text { between clinical } \\
\text { pharmacists and the } \\
\text { prescribers to } \\
\text { optimize prescribing: } \\
\text { auditing of the } \\
\text { physician's orders } \\
\text { and providing } \\
\text { feedback and } \\
\text { recommendations } \\
\text { during medical } \\
\text { rounds, reminders, } \\
\text { and discussions with } \\
\text { physicians }\end{array}$ & $\begin{array}{l}\text { Change in the incidence } \\
\text { rate of PIM }\end{array}$ & $\begin{array}{l}\text { Incidence rate of PIM } \\
\text { B: } 61 \% \text { vs. A: } 29.5 \% \text {, } \\
p<0.001\end{array}$ \\
\hline
\end{tabular}


TABLE 6 | (Continued) Effects of multifaceted interventions on inappropriate prescribing in older adults $(n=9)$.

\begin{tabular}{|c|c|c|c|c|c|c|c|c|}
\hline \multirow{2}{*}{$\begin{array}{l}\text { Author } \\
\text { (year) }\end{array}$} & \multicolumn{2}{|c|}{ Educational } & \multirow[t]{2}{*}{ CDSS } & \multirow{2}{*}{$\begin{array}{c}\text { Medication } \\
\text { review }\end{array}$} & \multirow{2}{*}{$\begin{array}{c}\text { PIM screening } \\
\text { tool }\end{array}$} & \multirow[t]{2}{*}{ Strategies used } & \multirow[t]{2}{*}{ Outcome measures } & \multirow{2}{*}{$\begin{array}{l}\text { Significant } \\
\text { outcomes }\end{array}$} \\
\hline & Performed by & Receivers & & & & & & \\
\hline $\begin{array}{l}\text { Gibert et al. } \\
(2018)\end{array}$ & Research team & 20 Phys & & Phys & STOPP criteria & $\begin{array}{l}\text { STOPP criteria use } \\
\text { during primary care } \\
\text { GP consultations }\end{array}$ & $\begin{array}{l}\text { Proportion of patients } \\
\text { with a reduction of PIM } \\
\text { after the intervention }\end{array}$ & $\begin{array}{l}\text { This intervention } \\
\text { reduced PIM for } \\
44.9 \% \text { of the patients } \\
(n=44), p<0.001\end{array}$ \\
\hline $\begin{array}{l}\text { Vandenberg } \\
\text { et al. (2018) }\end{array}$ & $\begin{array}{l}\text { Geriatrician, } \\
\text { Pharm, } \\
\text { Gerontologist }\end{array}$ & $\begin{array}{l}20 \text { primary } \\
\text { care } \\
\text { providers, } 4 \\
\text { Pharms }\end{array}$ & & Pharm & $\begin{array}{l}2012 \text { Beers } \\
\text { criteria }\end{array}$ & $\begin{array}{l}\text { A pharmacist-led, } \\
\text { one-on-one } \\
\text { medication review, to } \\
\text { provide rural primary } \\
\text { care providers and } \\
\text { pharmacists with } \\
\text { educational outreach } \\
\text { through academic } \\
\text { detailing and tools to } \\
\text { support safe geriatric } \\
\text { prescribing } \\
\text { practices, as well as } \\
\text { individual audit and } \\
\text { feedback on } \\
\text { prescribing practice } \\
\text { and confidential peer } \\
\text { benchmarking }\end{array}$ & $\begin{array}{l}\text { PIM incidence: number } \\
\text { of new PIM } \\
\text { prescriptions divided by } \\
\text { all encounters } \\
\text { (opportunities) that a } \\
\text { provider had with } \\
\text { veterans aged } 65 \text { years } \\
\text { and older; PIM } \\
\text { prevalence-number of } \\
\text { encounters with } \\
\text { veterans currently } \\
\text { taking at least } 1 \text { PIM } \\
\text { divided by all } \\
\text { encounters; multiple } \\
\text { PIM } \\
\text { prevalence-number of } \\
\text { encounters with } \\
\text { veterans taking } 2 \text { or } \\
\text { more PIM divided by all } \\
\text { encounters }\end{array}$ & $\begin{array}{l}\text { the intervention, } \\
\text { reaching significance } \\
(p=0.009) \text { during } \\
\text { the postintervention } \\
\text { period. PIM } \\
\text { prevalence declined } \\
\text { at baseline, } 22.6 \% \\
\text { encounters per } \\
\text { month were with } \\
\text { older veterans taking } \\
\text { at least } 1 \text { PIM. After } \\
\text { the intervention, this } \\
\text { proportion had } \\
\text { dropped to } 16.7 \% \\
\text { ( } p<0.001), \text { a } 26 \% \\
\text { relative reduction. } \\
\text { Encounters with } \\
\text { veterans taking } 2 \text { or } \\
\text { more PIM showed } \\
\text { steady and significant } \\
\text { decline, from a } \\
\text { baseline of } 6.2-4.1 \% \\
\text { after the intervention } \\
(p<0.001), \\
\text { representing a } 33.9 \% \\
\text { relative reduction in } \\
\text { multiple PIM } \\
\text { prevalence }\end{array}$ \\
\hline $\begin{array}{l}\text { Moss et al. } \\
\text { (2016) }\end{array}$ & $\begin{array}{l}\text { Phys, } \\
\text { Geriatricians, } \\
\text { Gerontologists, } \\
\text { Pharm, N, } \\
\text { Clinical } \\
\text { application } \\
\text { coordinators }\end{array}$ & $\begin{array}{l}73 \text { ED } \\
\text { providers (10 } \\
\text { physicians, } \\
60 \text { medical } \\
\text { residents), } 3 \\
\text { advanced } \\
\text { practice } \\
\text { providers }\end{array}$ & $x$ & & $\begin{array}{l}2012 \text { Beers } \\
\text { criteria }\end{array}$ & $\begin{array}{l}\text { Provider education; } \\
\text { clinical decision } \\
\text { support, and } \\
\text { provider feedback on } \\
\text { prescribing practices }\end{array}$ & $\begin{array}{l}\text { Rate of PIM prescribing } \\
\text { over the observation } \\
\text { period }\end{array}$ & Not achieved \\
\hline $\begin{array}{l}\text { Clyne et al. } \\
\text { (2015) }\end{array}$ & Pharm & $\begin{array}{l}\text { Phys (C: 11, } \\
\text { I: 10) }\end{array}$ & & Phys & $\begin{array}{l}\text { Beers, STOPP, } \\
\text { McLeod, IPET, } \\
\text { ACOVE, and } \\
\text { the Prescription } \\
\text { Peer Academic } \\
\text { Detailing } \\
\text { (RxPAD) } \\
\text { study-MRC } \\
\text { framework }\end{array}$ & $\begin{array}{l}\text { Academic detailing, } \\
\text { review of medicines } \\
\text { with web-based } \\
\text { pharmaceutical } \\
\text { treatment algorithms } \\
\text { that provide } \\
\text { recommended } \\
\text { alternative-treatment } \\
\text { options, and tailored } \\
\text { patient information } \\
\text { leaflets }\end{array}$ & Mean number of PIM & $\begin{array}{l}\text { Mean number of PIM } \\
\text { (SD) at baseline } \\
\text { C: } 1.39(0.6) \text { vs. I: } \\
1.31(0.6) \\
\text { Mean number of PIM } \\
\text { (SD) after intervention } \\
\text { completed } \\
\text { C: } 1.18(0.1) \text { vs. I: } \\
0.70(0.1), p=0.02\end{array}$ \\
\hline
\end{tabular}

A, after group; B, before group; C, control group; CDSS, clinical decision support system; CI, confidence interval; ED, emergency department; GP, general practitioner; I, intervention group; N, nurse; Pharm, pharmacist; Phys, physician; PIM, potentially inappropriate medication; SD, standard deviation; STRIP, systematic tool to reduce inappropriate prescribing; STOPP, Screening Tool of Older People's potentially inappropriate Prescriptions.

being prescribed one or more PIM (Urfer et al., 2016). Finally, one study observed that although the total number of newly prescribed PIM did not decrease, the top 10 most common new PIM significantly decreased from $9.0 \%$ to $8.3 \%$ (Vanderman et al., 2017).

\subsubsection{Impact of Multifaceted Interventions}

Nine studies used a multifaceted approach as a strategy to decrease the number of PIM (Clyne et al., 2016; Moss et al., 2016; Gibert et al., 2018; Najjar et al., 2018; Vandenberg et al., 2018; Boersma et al., 2019; Liu et al., 2019; Vu and Huong, 2019; 
TABLE 7 | Effects of organizational interventions ${ }^{1}$ on inappropriate prescribing in older adults $(n=2)$.

\begin{tabular}{|c|c|c|c|c|}
\hline $\begin{array}{l}\text { Author } \\
\text { (year) }\end{array}$ & PIM screening tool & Strategies used & $\begin{array}{l}\text { Outcome } \\
\text { measures }\end{array}$ & Significant outcomes \\
\hline $\begin{array}{l}\text { Stevens et al. } \\
(2017)\end{array}$ & 2012 Beers criteria & $\begin{array}{l}\text { Education, informatics-based clinical decision support } \\
\text { designed for improved workflow, and individual provider } \\
\text { feedback }\end{array}$ & $\begin{array}{l}\text { Average percentage } \\
\text { of PIM }\end{array}$ & $\begin{array}{l}\text { Average percentage of PIM per month (SD): } \\
\text { Site } 1-\text { B: } 11.9 \%(1.8) \text { vs A: } 5.1 \%(1.5) \\
p<0.0001 \\
\text { Site } 2-\text { B: } 8.2 \%(0.8) \text { vs A: } 4.5 \%(1.0) \\
p<0.0001 \\
\text { Site } 3-\text { B: } 8.9 \%(1.9) \text { vs A: } 6.1 \%(1.7) \\
p=0.0007 \\
\text { Site } 4-\text { B: } 7.4 \%(1.7) \text { vs A: } 5.7 \%(0.8) \\
p=0.04\end{array}$ \\
\hline $\begin{array}{l}\text { Wessell et al. } \\
\text { (2008) }\end{array}$ & $\begin{array}{l}1997 \text { Beers criteria (US } \\
2003 \text { update) }\end{array}$ & A quarterly PIM performance report; biannual on-site visits & $\begin{array}{l}\text { Change in the } \\
\text { prescription rate }\end{array}$ & $\begin{array}{l}\text { Absolute annual decline of } 0.018 \% \text { for } \\
\text { always inappropriate medications }(p=0.03)\end{array}$ \\
\hline
\end{tabular}

A, After group; B, Before group; PIM, Potentially inappropriate medication; SD, Standard deviation; US, United States.

${ }^{1}$ Organizational intervention- a combination of strategies to improve the quality indicators of institutions/organizations and enrolled in the approach all stakeholders, health professionals, and non-health professionals. This intervention uses several approaches, including diagnostic activity (including medication review), Team Building, Intergroup relationship, sensitivity training (including educational sessions.

Akkawi et al., 2020) In two studies, the multifaceted approach consisted in the application of a CDSS followed by a medication review (Boersma et al., 2019; Liu et al., 2019). One study used a combination of educational and CDSS approaches (Akkawi et al., 2020). The remaining studies used, as an approach to decrease the number of PIM, a combination of an educational approach followed by a medication review (Clyne et al., 2016; Moss et al., 2016; Gibert et al., 2018; Najjar et al., 2018; Vandenberg et al., 2018; Vu and Huong, 2019).

\section{Clinical Decision Support System and Medication Review}

The combined use of CDSS and medication review strategies led to a significant reduction of the mean number of PIM per patient from 0.7 to 0.23 (Boersma et al., 2019) and an increasing number of PIM changes implementation (intervention: $46.2 \%$ vs. control: 15.3\%) (Liu et al., 2019).

\section{Educational Intervention and Medication Review}

Five of the six studies that used a combination of educational and medication review strategies observed a significant impact on the PIM reduction (Clyne et al., 2016; Gibert et al., 2018; Najjar et al., 2018; Vandenberg et al., 2018; Vu and Huong, 2019). Among these, two studies reported a significant decrease in the mean number of PIM per patient from $0.99-1.18$ before intervention to 0.66 to 0.7, after intervention (Clyne et al., 2015; Gibert et al., 2018). Vandenberg et al. (2018) and Vu and Huong (2019) reported that the prevalence of PIM decreased 5.9\% and 10.9\%, respectively. According to Najjar et al. (2018), the multifaceted approach led to a decrease in the PIM incidence of $31.5 \%$.

\section{Educational Intervention and Clinical Decision Support System}

In one study (Akkawi et al., 2020), the multifaceted intervention consists of three educational sessions about PIM and discussion of STOPP criteria, coupled with an introduction of a CDSS. This approach did not achieve significant outcomes.

\subsubsection{Impact of Organizational Interventions}

One study performed an organizational intervention in four different hospitals and observed a significant decrease in the average percentage of prescribed PIM per month (1.7-6.8) after intervention (Stevens et al., 2017). Another study performed in 99 primary care practices observed that the organizational intervention that includes PIM performance reports, on-site visits, and network meetings was responsible for an absolute annual decline of $0.018 \%$ for always inappropriate medication (Wessell et al., 2008).

\subsubsection{Impact of Interventions That Have Greater Evidence by Its Design}

The analysis of the studies that included a concurrent control and low risk of bias revealed that in the hospital, all the five medication review interventions were effective (Spinewine et al., 2007; Gallagher et al., 2011; Dalleur et al., 2014; Van der Linden et al., 2017; Van Der Linden et al., 2018), two CDSS interventions were effective (Urfer et al., 2016; McDonald et al., 2019), one multifaceted intervention achieved significant impact (Vandenberg et al., 2018), and none of the educational interventions achieved a successful reduction of PIM. In primary care it was observed that all the two multifaceted interventions achieved a significant reduction of PIM (Clyne et al., 2015; Boersma et al., 2019), one (Clyne et al., 2016) of two educational strategies was effective, and none of the medication review and CDSS strategies was well successful.

\section{DISCUSSION}

Despite the extensive number of studies in the literature on PIM in older patients, only 31 of the included studies reported effective intervention. Among these, 21 presented methodological intervention limitations and could not ensure that the intervention used can be replicated and identical outcomes achieved (Brown and Earnhart, 2004; Fick et al., 2004; Spinewine et al., 2007; Wessell et al., 2008; Gallagher et al., 
2011; Dalleur et al., 2014; Frankenthal et al., 2014; Clyne et al., 2015; Tallon et al., 2015; Urfer et al., 2016; Frankenthal et al., 2017; Van der Linden et al., 2017; Etxeberria et al., 2018; Gibert et al., 2018; Hurmuz et al., 2018; Van Der Linden et al., 2018; Boersma et al., 2019; Gutiérrez-Valencia et al., 2019; Khera et al., 2019; Liu et al., 2019; Vu and Huong, 2019). Although a metaanalysis was not done, our findings suggested that in the hospital, the most adequate strategy to decrease the number of PIM and/or the patients with at least one PIM was medication review. Concerning primary care setting, the analysis of all the included studies indicated that educational interventions were the most successful. However, when only randomized controlled trial (RCT) studies were analyzed, it did not find greater effectiveness of some interventions over others.

The data of this study also suggested that the inclusion of pharmacists can upgrade the quality of the PIM intervention and effectively promote the well-being of the patients.

Regarding the influence of the number of prescribed medicines per patient in PIM interventions, our data suggested that the success of an intervention is not medicines number-dependent, since the analysis of the successful intervention rate in polymedicated and non-polymedicated patients was similar $(\approx 67 \%)$.

This work also suggested that most of the studies presented important design limitations, something that limits the grade of their evidence.

Medication review was the most frequent strategy used to improve pharmacotherapy and reduce the number of PIM in hospitalized patients. A reduction in the number of PIM per patient or/and in the number of patients with at least a PIM was achieved for $75 \%$ of the medication review interventions (Brown and Earnhart 2004; Spinewine et al., 2007; Gallagher et al., 2011; Keith et al., 2013; Dalleur et al., 2014; Ilić et al., 2015; Tallon et al., 2015; Van der Linden et al., 2017; Chan et al., 2018; Fajreldines et al. 2018; Van Der Linden et al., 2018; Gutiérrez-Valencia et al., 2019). Among the three studies that do not have efficacy in hospitalized patients, the main reasons pointed were as follows: 1) the difficulty to engage physicians to actively participate in the study - they preferred to receive the documentation about drug therapy issues by paper instead of discussing face-to-face the patients' pharmacotherapy (Chan et al., 2018); and 2) the low acceptance of the recommendation by the physicians (Regueiro et al., 2019).

In primary care, $42.9 \%$ of the interventional studies (Keith et al., 2013; Hurmuz et al., 2018; Stuckey et al., 2018; Khera et al., 2019) used medication review to improve the pharmacotherapy through the reduction of PIM. The lack of efficacy can be related to 1) the low acceptance rate of recommendations by the physicians (Allard et al., 2001); 2) the PIM list used-for example, Castelino et al. reported that in Australia the medicines listed in Beers criteria were rarely used; 3) the physicians did not use routinely any checklist and do not access computer programs to evaluate hypothetical interactions, and they do not record short-term drug alterations (Lampela et al., 2010); 4) in some cases the patients did not receive the full intervention (Allard et al., 2001); and 6) contamination between control and intervention groups (Campins et al., 2016).

The analysis of all educational interventions performed in primary care revealed that this type of intervention has been successfully implemented in $75 \%$. However, only one of the two studies that have greater evidence by its design effectively decreased the number of PIM. The success of educational interventions in primary care can be related to the promotion of a specific web training on PIM tools used by physicians in clinical practice, updating the knowledge of physicians in PIM detection (Fick et al., 2004; Clyne et al., 2016; Etxeberria et al., 2018). The lack of efficacy of educational intervention observed in one study can be related to a change in the participants' behavior due to the knowledge that they are taking part in an experiment (Hawthorne effect) (Rognstad et al., 2013).

In hospitalized patients, the poor outcomes achieved by educational interventions can be related to the low interactivity during the education intervention, the lack of knowledge of the clinicians, and the characteristics of the ward included that sometimes make difficult the collection of the data (Franchi et al., 2016).

The implementation of a CDSS in hospitals had a positive impact on 50\% of the studies (Urfer et al., 2016; Vanderman et al., 2017). The lack of efficacy of the intervention in the remaining studies can be related to the study design and the fact that the applied criteria are not setting-directed originating a high number of alerts that tend to be ignored by a healthcare professional (McDonald et al., 2019; Winata et al., 2020).

Multifaceted interventions were described as mixed interventions that can reduce the number of PIM (Rahme et al., 2005). In the hospital setting, it was observed that in the two studies that used a combination of educational and medication review strategies, the intervention was well successful (Najjar et al., 2018; Vu and Huong, 2019). In primary care, a combination of educational and medication review strategies results in increased efficacy of the intervention (Clyne et al., 2015; Gibert et al., 2018; Vandenberg et al., 2018).

The results of the included studies suggested that medication review is the most indicated intervention to promote the wellbeing of the hospitalized patients through the reduction of PIM. The success of medication review strategies at hospital discharge could be related to the fact that the inpatient setting may predispose older adults to new prescriptions and probably unnecessary drugs (Page et al., 2010). Moreover, during the hospitalization physicians tend to resist the change or discontinuation of chronic medication, particularly if the medication is not related to the reason for hospitalization (Page et al., 2010). This high number of prescribed PIM during the hospitalization can be the result of the lack of implemented PIM programs directed to each hospital ward and/or specific condition (Motter et al., 2018).

To improve the well-being of older adults, besides strategies to reduce PIM, strategies to promote appropriate prescription have also been developed. Medication review is a widely used strategy and with better outcomes to reduce potentially inappropriate prescribing (PIP) in hospitalized older adults. However, in a recent review, Dautzenberg et al. (2021) reported that the heterogeneity between studies does not allow reaching significant conclusion. According to dos Santos et al. (2019), the choice of outcome measures, study design, and 
methodological quality of medication review studies make it difficult to analyze the effectiveness of this strategy. The failure of medication review strategies in primary care can be attributed to the lack of time of physicians to perform the medication review (Plácido et al., 2020); also, as a result of this lack of time, even when the medication review was performed, patients' follow-up did not occur (Campins et al., 2016). On the other hand, educational strategies allow the empowerment of primary care physicians who already had enough handling in managing older adults but not the right confidence and knowledge to manage PIM prescription (Maio et al., 2011). Moreover, educational strategies had more impact on prescribing patterns than presenting a physician only with a decision algorithm (Rahme et al., 2005). A previous work observed similar results regarding the effectiveness of educational strategies to reduce PIP in primary care. According to Kunstler et al. (2019), educational strategies are well successful in changing health professional prescribing behavior.

A recent systematic review focused on non-clinical programs to reduce the inappropriate or unnecessary use of medicines observed that interventions consisting of education messages and recommended behavior alternatives were more likely to be successful in reducing the inappropriate use of medicines or medical procedures (Lin et al., 2020). Educational strategies are essential to improve prescription, as observed by Amorim et al. (2021) since physician-related characteristics can influence the number of PIM prescriptions.

Regarding the multifaceted strategies, the scarcity of studies using this approach did not allow clarifying their benefits in PIM reduction.

In the hospital setting, CDSS interventions significantly reduced the PIM number in older adults. Similar results were found in another systematic review (Dalton et al., 2018). The lack of CDSS in primary care can be related to the outdated user interface model (Price et al., 2017).

Regarding the organizational strategies, the studies achieved a significant impact on pharmacotherapy independently of the setting (Wessell et al., 2008; Stevens et al., 2017).

In $47.8 \%$ of the included studies, the intervention was performed by a pharmacist or by a multifaceted team that includes at least one pharmacist. Among these studies, the rate that interventions succeeded well was $72.7 \%$. In the remaining studies, the rate of success observed was $62.5 \%$, suggesting that the inclusion of a pharmacist in the PIM interventions team can be beneficial. Previously it was demonstrated that pharmacists are actively engaged in several care-delivery models such as direct patients care and collaborative team-based care, improving pharmacotherapy and ameliorating the patients-related health outcomes (Lee et al., 2015). It was also reported that pharmacists could play an important role in patients' medication review in practice settings such as community pharmacies long-term care facilities, outpatient clinic home care, and hospitals. Moreover, pharmacists-led deprescribing interventions can reduce the number of unnecessary and potentially harmful medications (Silva et al., 2019; Hernández-Prats et al., 2021).

Although this study was performed with scientific rigors, some limitations are present. The search strategy was limited to the three main health research databases and articles written in English, Portuguese, and Spanish. The included studies were heterogeneous in practice settings, population, size of the samples, and PIM definition that can be variable depending on the screening tool used, which can influence PIM number detected (Thomas and Thomas, 2019; Perpétuo et al., 2021).

Because this review includes studies independently of the quality assessment analysis, an outcomes bias can be aroused. The bias can be attributed to a lack of randomization and blinded interventions and absences/inadequate follow-up period in some studies, compromising a possible scaling up of the interventions.

This study provided valuable data regarding PIM-reduction strategies; however, most of the included studies presented limitations that restrain the extrapolation of the results and a lack of an economic evaluation. Only one study reported that in the intervention group, a significantly lower medication cost was achieved (Frankenthal et al., 2014).

A recent systematic review only found seven articles reporting the economic impact of PIM interventions and suggested that although limited, interventions to optimize medication may outweigh their implementation costs (Laberge et al., 2021).

\section{DATA AVAILABILITY STATEMENT}

The original contributions presented in the study are included in the article/Supplementary Material, further inquiries can be directed to the corresponding author.

\section{AUTHOR CONTRIBUTIONS}

All authors meet all four ICJME criteria for authorship. Conceptualization: FR and MH; Data curation: DR, AP, FR, $\mathrm{MH}$, and AF; Formal analysis: DR and AP; Funding acquisition: FR and MH; Investigation: AP and DR; Methodology: FR and MH; Project administration: FR; Resources: FR; Supervision: FR, MH, RM-C, and AF; Validation: FR, AF, and MT-H; Roles/ Writing-original draft: AP and DR; Writing-review and editing: FR, MH, RM-C, and AF.

\section{FUNDING}

This work was financially supported by the project APIMedOlder (PTDC/MED-FAR/31598/2017), funded by FEDER, through COMPETE2020-Programa Operacional Competitividade e Internacionalização (POCI-01-0145-FEDER-031598) and by national funds (OE), through FCT/MCTES.

\section{SUPPLEMENTARY MATERIAL}

The Supplementary Material for this article can be found online at: https://www.frontiersin.org/articles/10.3389/fphar.2021.777655/ full\#supplementary-material 


\section{REFERENCES}

Akkawi, M. E., Nik Mohamed, M. H., and Md Aris, M. A. (2020). The Impact of a Multifaceted Intervention to Reduce Potentially Inappropriate Prescribing Among Discharged Older Adults: A Before-And-After Study. J. Pharm. Pol. Pract. 13, 39. doi:10.1186/s40545-020-00236-0

Allard, J., Hébert, R., Rioux, M., Asselin, J., and Voyer, L. (2001). Efficacy of a Clinical Medication Review on the Number of Potentially Inappropriate Prescriptions Prescribed for Community-Dwelling Elderly People. CMAJ $164,1291-1296$.

Amorim, W. W., Passos, L. C., Gama, R. S., Souza, R. M., Graia, L. T., Macedo, J. C., et al. (2021). Physician and Patient-Related Factors Associated with Inappropriate Prescribing to Older Patients within Primary Care: A CrossSectional Study in brazil. Sao Paulo Med. J. 139, 107-116. doi:10.1590/15163180.2020.0411.r1.18112020

Anderson, G. M., Beers, M. H., and Kerluke, K. (1997). Auditing Prescription Practice Using Explicit Criteria and Computerized Drug Benefit Claims Data. J. Eval. Clin. Pract. 3, 283-294. doi:10.1046/j.1365-2753.1997.t01-100005.x

Barnett, K., Mercer, S. W., Norbury, M., Watt, G., Wyke, S., and Guthrie, B. (2012). Epidemiology of Multimorbidity and Implications for Health Care, Research, and Medical Education: A Cross-Sectional Study. Lancet 380, 37-43. doi:10.1016/S0140-6736(12)60240-2

Beard, J. R., Officer, A., De Carvalho, I. A., Sadana, R., Pot, A. M., Michel, J. P., et al. (2016). The World Report on Ageing and Health: A Policy Framework for Healthy Ageing. Lancet 387, 2145-2154. doi:10.1016/S0140-6736(15)00516-4

Bhugra, D., and Cutter, W. (2001). "Mentally Ill: Public Attitudes," in International Encyclopedia of the Social \& Behavioral Sciences. Editors N. J. Smelser and P. B. Baltes (Oxford: Pergamon Press), 9704-9709. doi:10.1016/b0-08-0430767/03701-3

Boersma, M. N., Huibers, C. J. A., Drenth-van Maanen, A. C., Emmelot-Vonk, M. H., Wilting, I., and Knol, W. (2019). The Effect of Providing Prescribing Recommendations on Appropriate Prescribing: A Cluster-Randomized Controlled Trial in Older Adults in a Preoperative Setting. Br. J. Clin. Pharmacol. 85, 1974-1983. doi:10.1111/bcp.13987

Brown, B. K., and Earnhart, J. (2004). Pharmacists and Their Effectiveness in Ensuring the Appropriateness of the Chronic Medication Regimens of Geriatric Inpatients. Consult. Pharm. 19, 432-436. doi:10.4140/TCP.n.2004.432

Cady, S., and Kim, J. (2017). What We Can Learn from Evaluating OD Interventions. Organizational and Individual Change. 49 (1), 50-54. doi:10.24247/ijhrmrdec20171

Campins, L., Serra-Prat, M., Gózalo, I., López, D., Palomera, E., Agustí, C., et al. (2016). Randomized Controlled Trial of an Intervention to Improve Drug Appropriateness in Community-Dwelling Polymedicated Elderly People. Fam. Pract. 34, 36-42. doi:10.1093/fampra/cmw073

Castelino, R. L., Hilmer, S. N., Bajorek, B. V., Nishtala, P., and Chen, T. F. (2010). Drug Burden Index and Potentially Inappropriate Medications in CommunityDwelling Older People: The Impact of home Medicines Review. Drugs Aging 27, 135-148. doi:10.2165/11531560-000000000-00000

Chan, W. W. T., Dahri, K., Partovi, N., Egan, G., and Yousefi, V. (2018). Evaluation of Collaborative Medication Reviews for High-Risk Older Adults. Can. J. Hosp. Pharm. 71, 356-363. doi:10.4212/cjhp.v71i6.2850

Clyne, B., Smith, S. M., Hughes, C. M., Boland, F., Bradley, M. C., Cooper, J. A., et al. (2015). Effectiveness of a Multifaceted Intervention for Potentially Inappropriate Prescribing in Older Patients in Primary Care: A ClusterRandomized Controlled Trial (OPTI-SCRIPT Study). Ann. Fam. Med. 13, 545-553. doi:10.1370/afm. 1838

Clyne, B., Smith, S. M., Hughes, C. M., Boland, F., Cooper, J. A., and Fahey, T. (2016). Sustained Effectiveness of a Multifaceted Intervention to Reduce Potentially Inappropriate Prescribing in Older Patients in Primary Care (OPTI-SCRIPT Study). Implement. Sci. 11, 79. doi:10.1186/s13012-016-0442-2

Dalleur, O., Boland, B., Losseau, C., Henrard, S., Wouters, D., Speybroeck, N., et al. (2014). Reduction of Potentially Inappropriate Medications Using the STOPP Criteria in Frail Older Inpatients: A Randomised Controlled Study. Drugs Aging 31, 291-298. doi:10.1007/s40266-014-0157-5

Dalton, K., O'Brien, G., O'Mahony, D., and Byrne, S. (2018). Computerised Interventions Designed to Reduce Potentially Inappropriate Prescribing in
Hospitalised Older Adults: A Systematic Review and Meta-Analysis. Age Ageing 47, 670-678. doi:10.1093/ageing/afy086

Dautzenberg, L., Bretagne, L., Koek, H. L., Tsokani, S., Zevgiti, S., Rodondi, N., et al. (2021). Medication Review Interventions to Reduce Hospital Readmissions in Older People. J. Am. Geriatr. Soc. 69, 1646-1658. doi:10.1111/jgs.17041

dos Santos, N. S., Marengo, L. L., Moraes, F. D. S., and Barberato Filho, S. (2019). Interventions to Reduce the Prescription of Inappropriate Medicines in Older Patients. Rev. Saude Publica 53, 7. doi:10.11606/S15188787.2019053000781

Etxeberria, A., Iribar, J., Rotaeche, R., Vrotsou, K., Barral, I., Barral, I., et al. (2018). Evaluation of an Educational Intervention and a Structured Review of Polypharmacy in Elderly Patients in Primary Care. Rev. Esp. Geriatr. Gerontol. 53, 319-325. doi:10.1016/j.regg.2018.07.002

Fajreldines, A., Schnitzler, E., Insua, J. T., Valerio, M., Davide, L., and Pellizzari, M. (2018). Reducción de Prescripción Inapropiada y Eventos Adversos a Medicamentos en Ancianos Hospitalizados [Reduction of inappropriate Prescriptions and Adverse Effects to Medications in Hospitalized Elderly Patients]. Medicina (B. Aires), 78 (1), 11-17. Spanish.

Fick, D. M., Maclean, J. R., Rodriguez, N. A., Short, L., Heuvel, R. V., Waller, J. L., et al. (2004). A Randomized Study to Decrease the Use of Potentially Inappropriate Medications Among Community-Dwelling Older Adults in a southeastern Managed Care Organization. Am. J. Manag. Care 10, 761-768.

Franchi, C., Mari, D., Tettamanti, M., Pasina, L., Djade, C. D., Mannucci, P. M., et al. (2014). E-Learning to Improve the Drug Prescribing in the Hospitalized Elderly Patients: The ELICADHE Feasibility Pilot Study. Aging Clin. Exp. Res. 26, 435-443. doi:10.1007/s40520-013-0187-6

Franchi, C., Tettamanti, M., Djade, C. D., Pasina, L., Mannucci, P. M., Onder, G., et al. (2016). E-Learning in Order to Improve Drug Prescription for Hospitalized Older Patients: A Cluster-Randomized Controlled Study. Br. J. Clin. Pharmacol. 82, 53-63. doi:10.1111/bcp.12922

Frankenthal, D., Israeli, A., Caraco, Y., Lerman, Y., Kalendaryev, E., ZandmanGoddard, G., et al. (2017). Long-Term Outcomes of Medication Intervention Using the Screening Tool of Older Persons Potentially Inappropriate Prescriptions Screening Tool to Alert Doctors to Right Treatment Criteria. J. Am. Geriatr. Soc. 65, e33. doi:10.1111/jgs.14570

Frankenthal, D., Lerman, Y., Kalendaryev, E., and Lerman, Y. (2014). Intervention with the Screening Tool of Older Persons Potentially Inappropriate Prescriptions/Screening Tool to Alert Doctors to Right Treatment Criteria in Elderly Residents of a Chronic Geriatric Facility: A Randomized Clinical Trial. J. Am. Geriatr. Soc. 62, 1658-1665. doi:10.1111/jgs.12993

Gallagher, P. F., O'Connor, M. N., and O'Mahony, D. (2011). Prevention of Potentially Inappropriate Prescribing for Elderly Patients: A Randomized Controlled Trial Using STOPP/START Criteria. Clin. Pharmacol. Ther. 89, 845-854. doi:10.1038/clpt.2011.44

Gibert, P., Cabaret, M., Moulis, M., Bosson, J. L., Boivin, J. E., Chanoine, S., et al. (2018). Optimizing Medication Use in Elderly People in Primary Care: Impact of STOPP Criteria on Inappropriate Prescriptions. Arch. Gerontol. Geriatr. 75, 16-19. doi:10.1016/j.archger.2017.10.022

Griese-Mammen, N., Hersberger, K. E., Messerli, M., Leikola, S., Horvat, N., van Mil, J. W. F., et al. (2018). PCNE Definition of Medication Review: Reaching Agreement. Int. J. Clin. Pharm. 40, 1199-1208. doi:10.1007/s11096-0180696-7

Gutiérrez-Valencia, M., Izquierdo, M., Beobide-Telleria, I., Ferro-Uriguen, A., Alonso-Renedo, J., Casas-Herrero, Á., et al. (2019). Medicine Optimization Strategy in an Acute Geriatric Unit: The Pharmacist in the Geriatric Team. Geriatr. Gerontol. Int. 19, 530-536. doi:10.1111/ggi.13659

Hefner, G., Hahn, M., Toto, S., Hiemke, C., Roll, S. C., Wolff, J., et al. (2021). Potentially Inappropriate Medication in Older Psychiatric Patients. Eur. J. Clin. Pharmacol. 77, 331-339. doi:10.1007/s00228-020-03012-w

Hernández-Prats, C., López-Pintor, E., and Lumbreras, B. (2021). Pharmacist-Led Intervention on the Reduction of Inappropriate Medication Use in Patients with Heart Failure: A Systematic Review of Randomized Trials and NonRandomized Intervention Studies. Res. Soc. Administrative Pharm. S1551-7411 (21), 00240. doi:10.1016/j.sapharm.2021.06.023

Hurmuz, M. Z. M., Janus, S. I. M., and van Manen, J. G. (2018). Changes in Medicine Prescription Following a Medication Review in Older High-Risk Patients with Polypharmacy. Int. J. Clin. Pharm. 40, 480-487. doi:10.1007/ s11096-018-0602-3 
Ilić, D., Bukumiric, Z., and Jankovic, S. (2015). Impact of Educational Intervention on Prescribing Inappropriate Medication to Elderly Nursing Homes Residents. Srp. Arh. Celok. Lek. 143, 174-179. doi:10.2298/SARH1504174I

Kaur, S., Mitchell, G., Vitetta, L., Roberts, M. S., and Gallagher, P. (2009). Interventions that Can Reduce Inappropriate Prescribing in the Elderly: A Systematic Review. Drugs Aging 26, 1013-1028. doi:10.2165/11318890000000000-00000

Keith, S. W., Maio, V., Dudash, K., Templin, M., and Del Canale, S. (2013). A Physician-Focused Intervention to Reduce Potentially Inappropriate Medication Prescribing in Older People: A 3-year, Italian, Prospective, Proof-Of-Concept Study. Drugs Aging 30, 119-127. doi:10.1007/s40266-012-0043-y

Khera, S., Abbasi, M., Dabravolskaj, J., Sadowski, C. A., Yua, H., and Chevalier, B. (2019). Appropriateness of Medications in Older Adults Living With Frailty: Impact of a Pharmacist-Led Structured Medication Review Process in Primary Care. J. Prim. Care Community Health 10, 2150132719890227. doi:10.1177/ 2150132719890227

Kunstler, B. E., Lennox, A., and Bragge, P. (2019). Changing Prescribing Behaviours with Educational Outreach: An Overview of Evidence and Practice. BMC Med. Educ. 19, 311. doi:10.1186/s12909-019-1735-3

Laberge, M., Sirois, C., Lunghi, C., Gaudreault, M., Nakamura, Y., Bolduc, C., et al. (2021). Economic Evaluations of Interventions to Optimize Medication Use in Older Adults with Polypharmacy and Multimorbidity: A Systematic Review. Clin. Interv. Aging 16, 767-779. doi:10.2147/CIA.S304074

Lampela, P., Hartikainen, S., Lavikainen, P., Sulkava, R., and Huupponen, R. (2010). Effects of Medication Assessment as Part of a Comprehensive Geriatric Assessment on Drug Use Over a 1-Year Period: A Population-Based Intervention Study. Drugs Aging 27, 507-521. doi:10.2165/11536650000000000-00000

Lee, J., Alshehri, S., Kutbi, H., and Martin, J. (2015). Optimizing Pharmacotherapy in Elderly Patients: the Role of Pharmacists. Integr. Pharm. Res. Pract. 4, 101-111. doi:10.2147/iprp.s70404

Lin, L., Alam, P., Fearon, E., and Hargreaves, J. R. (2020). Public Target Interventions to Reduce the Inappropriate Use of Medicines or Medical Procedures: A Systematic Review. Implement. Sci. 15, 90. doi:10.1186/ s13012-020-01018-7

Liu, Y. L., Chu, L. L., Su, H. C., Tsai, K. T., Kao, P. H., Chen, J. F., et al. (2019). Impact of Computer-Based and Pharmacist-Assisted Medication Review Initiated in the Emergency Department. J. Am. Geriatr. Soc. 67, 2298-2304. doi:10.1111/jgs. 16078

Lopatto, J., Keith, S. W., Del Canale, S., Templin, M., and Maio, V. (2014). Evaluating Sustained Quality Improvements: Long-Term Effectiveness of a Physician-Focused Intervention to Reduce Potentially Inappropriate Medication Prescribing in an Older Population. J. Clin. Pharm. Ther. 39, 266-271. doi:10.1111/jcpt.12137

Maio, V., Jutkowitz, E., Herrera, K., Abouzaid, S., Negri, G., and Del Canale, S. (2011). Appropriate Medication Prescribing in Elderly Patients: How Knowledgeable Are Primary Care Physicians? A Survey Study in Parma, Italy. J. Clin. Pharm. Ther. 36, 468-480. doi:10.1111/j.1365-2710.2010.01195.x

McDonald, E. G., Wu, P. E., Rashidi, B., Forster, A. J., Huang, A., Pilote, L., et al. (2019). The MedSafer Study: A Controlled Trial of an Electronic Decision Support Tool for Deprescribing in Acute Care. J. Am. Geriatr. Soc. 67, 1843-1850. doi:10.1111/jgs.16040

Moss, J. M., Bryan, W. E., Wilkerson, L. M., Jackson, G. L., Owenby, R. K., Van Houtven, C., et al. (2016). Impact of Clinical Pharmacy Specialists on the Design and Implementation of a Quality Improvement Initiative to Decrease Inappropriate Medications in a Veterans Affairs Emergency Department. J. Manag. Care Spec. Pharm. 22, 74-80. doi:10.18553/jmcp.2016.22.1.74

Moss, J. M., Bryan, W. E., Wilkerson, L. M., King, H. A., Jackson, G. L., Owenby, R. K., et al. (2019). An Interdisciplinary Academic Detailing Approach to Decrease Inappropriate Medication Prescribing by Physician Residents for Older Veterans Treated in the Emergency Department. J. Pharm. Pract. 32, 167-174. doi:10.1177/0897190017747424

Motter, F. R., Fritzen, J. S., Hilmer, S. N., Paniz, É. V., and Paniz, V. M. V. (2018). Potentially Inappropriate Medication in the Elderly: A Systematic Review of Validated Explicit Criteria. Eur. J. Clin. Pharmacol. 74, 679-700. doi:10.1007/ s00228-018-2446-0

Najjar, M. F., Sulaiman, S. A. S., Al Jeraisy, M., and Balubaid, H. (2018). The Impact of a Combined Intervention Program: an Educational and Clinical Pharmacist's
Intervention to Improve Prescribing Pattern in Hospitalized Geriatric Patients at King Abdulaziz Medical City in Riyadh, Saudi Arabia. Ther. Clin. Risk Manag. 14, 557-564. doi:10.2147/TCRM.S157469

National Heart Laboratory (2013). Study Quality Assessment Tools. Available at: https://www.nhlbi.nih.gov/health-topics/study-quality-assessment-tools (Accessed May 15, 2021).

Page, M. J., McKenzie, J. E., Bossuyt, P. M., Boutron, I., Hoffmann, T. C., Mulrow, C. D., et al. (2021). The PRISMA 2020 Statement: An Updated Guideline for Reporting Systematic Reviews. BMJ 372, n71. doi:10.1136/bmj.n71

Page, R. L., Linnebur, S. A., Bryant, L. L., and Ruscin, J. M. (2010). Inappropriate Prescribing in the Hospitalized Elderly Patient: Defining the Problem, Evaluation Tools, and Possible Solutions. Clin. Interv. Aging 5, 75-87. doi:10.2147/cia.s9564

Perpétuo, C., Plácido, A. I., Rodrigues, D., Aperta, J., Piñeiro-Lamas, M., Figueiras, A., et al. (2021). Prescription of Potentially Inappropriate Medication in Older Inpatients of an Internal Medicine Ward: Concordance and Overlap Among the EU(7)-PIM List and Beers and STOPP Criteria. Front. Pharmacol. 12, 676020. doi:10.3389/fphar.2021.676020

Plácido, A. I., Herdeiro, M. T., Simões, J. L., Amaral, O., Figueiras, A., and Roque, F. (2020). Voices of Polymedicated Older Patients: A Focus Group Approach. Int. J. Environ. Res. Public Health 17, 6443. doi:10.3390/ijerph17186443

Price, M., Davies, I., Rusk, R., Lesperance, M., and Weber, J. (2017). Applying Stopp Guidelines in Primary Care through Electronic Medical Record Decision Support: Randomized Control Trial Highlighting the Importance of Data Quality. JMIR Med. Inform. 5, e15. doi:10.2196/medinform.6226

Rahme, E., Choquette, D., Beaulieu, M., Bessette, L., Joseph, L., Toubouti, Y., et al. (2005). Impact of a General Practitioner Educational Intervention on Osteoarthritis Treatment in an Elderly Population. Am. J. Med. 118, 1262-1270. doi:10.1016/j.amjmed.2005.03.026

Regueiro, R., Estrada Menéndez, C., and Morís de la Tassa, J. (2019). Impacto de un programa de intervención destinado a la mejora de la prescripción potencialmente inapropiada en pacientes ancianos hospitalizados. Revista Clínica Española 219, 375-385. doi:10.1016/j.rce.2018.12.012

Renom-Guiteras, A., Meyer, G., and Thürmann, P. A. (2015). The EU(7)-PIM List: A List of Potentially Inappropriate Medications for Older People Consented by Experts from Seven European Countries. Eur. J. Clin. Pharmacol. 71, 861-875. doi:10.1007/s00228-015-1860-9

Rognstad, S., Brekke, M., Fetveit, A., Dalen, I., and Straand, J. (2013). Prescription Peer Academic Detailing to Reduce Inappropriate Prescribing for Older Patients: A Cluster Randomised Controlled Trial. Br. J. Gen. Pract. 63, e554-62. doi:10.3399/bjgp13X670688

Sennesael, A. L., Dalleur, O., Henrard, S., Artoisenet, C., Schoevaerdts, D., and Spinewine, A. (2018). Implementing a Screening Tool to Improve Prescribing in Hospitalized Older Patients: a Pilot Study. Int. J. Clin. Pharm. 40, 15-19. doi:10.1007/s11096-017-0563-y

Silva, R. O. S., Macêdo, L. A., Santos, G. A. D., Aguiar, P. M., and de Lyra, D. P. (2019). Pharmacist-Participated Medication Review in Different Practice Settings: Service or Intervention? An Overview of Systematic Reviews. PLoS One 14, e0210312. doi:10.1371/journal.pone.0210312

Simonson, W., and Feinberg, J. L. (2005). Medication-Related Problems in the Elderly : Defining the Issues and Identifying Solutions. Drugs Aging 22, 559-569. doi:10.2165/00002512-200522070-00002

Spinewine, A., Swine, C., Dhillon, S., Lambert, P., Nachega, J. B., Wilmotte, L., et al. (2007). Effect of a Collaborative Approach on the Quality of Prescribing for Geriatric Inpatients: A Randomized, Controlled Trial. J. Am. Geriatr. Soc. 55, 658-665. doi:10.1111/j.1532-5415.2007.01132.x

Squires, J. E., Sullivan, K., Eccles, M. P., Worswick, J., and Grimshaw, J. M. (2014). Are Multifaceted Interventions More Effective Than Single-Component Interventions in Changing Health-Care Professionals' Behaviours? An Overview of Systematic Reviews. Implement. Sci. 9, 152. doi:10.1186/s13012014-0152-6

Stevens, M., Hastings, S. N., Markland, A. D., Hwang, U., Hung, W., Vandenberg, A. E., et al. (2017). Enhancing Quality of Provider Practices for Older Adults in the Emergency Department (EQUiPPED). J. Am. Geriatr. Soc. 65, 1609-1614. doi:10.1111/jgs.14890

Stuckey, N., Henriksen, B., Singh, H., Dawson, A., and Waterson, Z. (2018). Interventions to Reduce High-Risk Medication Use in the Geriatric Population. Top. Geriatr. Rehabil. 34, 178-181. doi:10.1097/TGR.0000000000000191 
Tallon, M., Barragry, J., Allen, A., Breslin, N., Deasy, E., Moloney, E., et al. (2015). Impact of the Collaborative Pharmaceutical Care at Tallaght Hospital (PACT) Model on Medication Appropriateness of Older Patients. Eur. J. Hosp. Pharm. 23, 16-21. doi:10.1136/ejhpharm-2014-000511

Thomas, R. E., and Thomas, B. C. (2019). A Systematic Review of Studies of the STOPP/ START 2015 and American Geriatric Society Beers 2015 Criteria in Patients $\geq$ 65 Years. Curr. Aging Sci. 12, 121-154. doi:10.2174/1874609812666190516093742

United Nations (2019). World Population Prospects 2019: Highlights (ST/ESA/ SER.A/423). New York: Departement of Economic and Social Affairs.

Urfer, M., Elzi, L., Dell-Kuster, S., and Bassetti, S. (2016). Intervention to Improve Appropriate Prescribing and Reduce Polypharmacy in Elderly Patients Admitted to an Internal Medicine Unit. PLoS One 11, e0166359. doi:10.1371/journal.pone.0166359

Van der Linden, L., Decoutere, L., Walgraeve, K., Milisen, K., Flamaing, J., Spriet, I., et al. (2017). Combined Use of the Rationalization of Home Medication by an Adjusted STOPP in Older Patients (RASP) List and a Pharmacist-Led Medication Review in Very Old Inpatients: Impact on Quality of Prescribing and Clinical Outcome. Drugs Aging 34, 123-133. doi:10.1007/s40266-016-0424-8

Van Der Linden, L., Hias, J., Dreessen, L., Milisen, K., Flamaing, J., Spriet, I., et al. (2018). Medication Review Versus Usual Care to Improve Drug Therapies in Older Inpatients Not Admitted to Geriatric Wards: A Quasi-Experimental Study (RASP-IGCT). BMC Geriatr. 18, 155. doi:10.1186/s12877-018-0843-y

Vandenberg, A. E., Echt, K. V., Kemp, L., McGwin, G., Perkins, M. M., and Mirk, A. K. (2018). Academic Detailing with Provider Audit and Feedback Improve Prescribing Quality for Older Veterans. J. Am. Geriatr. Soc. 66, 621-627. doi:10.1111/jgs.15247

Vanderman, A. J., Moss, J. M., Bryan, W. E., Sloane, R., Jackson, G. L., and Hastings, S. N. (2017). Evaluating the Impact of Medication Safety Alerts on Prescribing of Potentially Inappropriate Medications for Older Veterans in an Ambulatory Care Setting. J. Pharm. Pract. 30, 82-88. doi:10.1177/ 0897190015621803
Vu, T. X., and Huong, Q. B. T. (2019). The Effect of the Pharmacist's Intervention on Potentially Inappropriate Medication Use in Older Adults Using the 2015 Beers Criteria. Pharm. Sci. Asia 46, 54-61. doi:10.29090/psa.2019.01.017.0023

Wessell, A. M., Nietert, P. J., Jenkins, R. G., Nemeth, L. S., and Ornstein, S. M. (2008). Inappropriate Medication Use in the Elderly: Results from a Quality Improvement Project in 99 Primary Care Practices. Am. J. Geriatr. Pharmacother. 6, 21-27. doi:10.1016/j.amjopharm.2008.02.001

Winata, S., Liacos, M., Crabtree, A., Page, A., and Moran, C. (2020). Electronic Medication Management System Introduction and Deprescribing Practice in Post-Acute Care. J. Am. Med. Dir. Assoc. 22, 90-95. doi:10.1016/j.jamda.2020.10.015

Conflict of Interest: The authors declare that the research was conducted in the absence of any commercial or financial relationships that could be construed as a potential conflict of interest.

Publisher's Note: All claims expressed in this article are solely those of the authors and do not necessarily represent those of their affiliated organizations or those of the publisher, the editors, and the reviewers. Any product that may be evaluated in this article, or claim that may be made by its manufacturer, is not guaranteed or endorsed by the publisher.

Copyright (c) 2022 Rodrigues, Plácido, Mateos-Campos, Figueiras, Herdeiro and Roque. This is an open-access article distributed under the terms of the Creative Commons Attribution License (CC BY). The use, distribution or reproduction in other forums is permitted, provided the original author $(s)$ and the copyright owner(s) are credited and that the original publication in this journal is cited, in accordance with accepted academic practice. No use, distribution or reproduction is permitted which does not comply with these terms. 